Communications in Mathematical Physics manuscript No.

(will be inserted by the editor)

\title{
Value distribution of the eigenfunctions and spectral determinants of quantum star graphs
}

\author{
J. P. Keating, J. Marklof and B. Winn \\ School of Mathematics, University of Bristol, Bristol. BS8 1TW, U.K. \\ E-mail: j.p.keating@bristol.ac.uk,j.marklof@bristol.ac.uk,b.winn@bristol.ac.uk \\ Received: 29 October 2002
}

\begin{abstract}
We compute the value distributions of the eigenfunctions and spectral determinant of the Schrödinger operator on families of star graphs. The values of the spectral determinant are shown to have a Cauchy distribution with respect both to averages over bond lengths in the limit as the wavenumber tends to infinity and to averages over wavenumber when the bond lengths are fixed and not rationally related. This is in contrast to the spectral determinants of random matrices, for which the logarithm is known to satisfy a Gaussian limit distribution. The value distribution of the eigenfunctions also differs from the corresponding random matrix result. We argue that the value distributions of the spectral determinant and of the eigenfunctions should coincide with those of Šeba-type billiards.
\end{abstract}

\section{Introduction}

The study of quantum graphs as model systems for quantum chaos was initiated by Kottos and Smilansky [19], 20], who observed that the spectral statistics of fully-connected graphs are typical of those associated with generic classically chaotic systems. The relative simplicity of quantum graphs, together with the existence of an exact trace formula, has lead to the suggestion that their study might provide insights into some of the fundamental problems of quantum chaos 18. This has motivated many works considering a variety of aspects of quantum graphs, 2], 5], 11], 12, 21], 24], 25, 26], 27], 30].

Studies 3], 4] of a special class of graphs - the so-called "hydra" graphs or star graphs - have revealed spectral statistics that are not typically associated with quantum chaotic systems. These have been dubbed "intermediate statistics" in recent works, 8 8, 9, 10] and have been observed in a number of systems. We are motivated to investigate this further by studying the value distributions of 
the eigenfunctions and the spectral determinant of the Schrödinger operator on quantum star graphs.

A star graph consists of a single central vertex together with $v$ outlying vertices each of which is connected only to the central vertex by a bond (figure 11). Hence there are $v$ bonds. We associate to each bond a length $L_{j}, j=1, \ldots, v$. We will often refer to the vector of bond lengths $\mathbf{L}:=\left(L_{1}, \ldots, L_{v}\right)$.

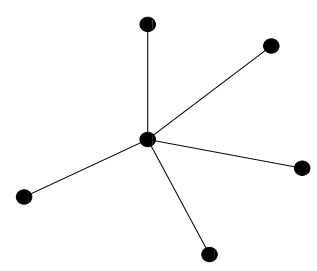

Fig. 1. A star graph with 5 bonds

The Schrödinger operator on a star graph takes the form of the Laplacian $-\mathrm{d}^{2} / \mathrm{d} x^{2}$ acting on the space of functions defined on the bonds of the graph that are twice-differentiable and satisfy the following matching conditions at the vertices:

$$
\begin{aligned}
\psi_{j}(0)=\psi_{i}(0) & =: \Psi, \quad j, i=1, \ldots, v \\
\sum_{j=1}^{v} \psi_{j}^{\prime}(0) & =\frac{1}{\lambda} \Psi \\
\psi_{j}^{\prime}\left(L_{j}\right) & =0, \quad j=1, \ldots, v .
\end{aligned}
$$

Here $\psi_{j}$ is the component of the function defined on the $j^{\text {th }}$ bond of the graph, and $\psi_{j}:\left[0, L_{j}\right] \rightarrow \mathbb{R}$ with the convention that $\psi_{j}(0)$ is the value of the function at the central vertex of the star graph. $\lambda$ is a parameter that allows us to vary the boundary conditions at the central vertex.

The Schrödinger operator so-defined is self-adjoint, so there exists a discrete unbounded set of values $0 \leq k_{0}<k_{1} \leq k_{2} \leq \cdots \rightarrow \infty$ such that $k_{n}^{2}$ is an eigenvalue. It can be shown that $k= \pm k_{n}$ corresponds to an eigenvalue if and only if it is a solution of $Z(k, \mathbf{L})=0$, where

$$
Z(k, \mathbf{L}):=\sum_{j=1}^{v} \tan k L_{j}-\frac{1}{k \lambda} .
$$

We refer to $Z(k, \mathbf{L})$ as the spectral determinant. Note that $Z(k, \mathbf{L})$ has poles at $k=(2 n+1) \pi / 2 L_{j}$ for each $n \in \mathbb{Z}$ and $j=1, \ldots, v$. The zeros and poles of $Z(k, \mathbf{L})$ interlace. The usual definition of the spectral determinant would require these poles to be factored out.

For simplicity we henceforth consider the case $1 / \lambda=0$. We shall employ the notation

$$
Z^{\prime}(k, \mathbf{L})=\frac{\partial Z}{\partial k}(k, \mathbf{L})=\sum_{j=1}^{v} L_{j} \sec ^{2} k L_{j} .
$$


The eigenfunction corresponding to the $n^{\text {th }}$ eigenvalue is found to be

$$
\psi_{i}^{(n)}(x)=A^{(n)} \frac{\cos k_{n}\left(x-L_{i}\right)}{\cos k_{n} L_{i}} .
$$

The constant $A^{(n)}$ is determined by the normalisation

$$
\sum_{j=1}^{v} \int_{0}^{L_{j}}\left|\psi_{j}^{(n)}(x)\right|^{2} \mathrm{~d} x=1
$$

to be

$$
A^{(n)}=\left(\frac{2}{\sum_{j=1}^{v} L_{j} \sec ^{2} k_{n} L_{j}}\right)^{\frac{1}{2}}
$$

The value distribution of the eigenfunctions is determined by these normalisation constants. For definiteness, we shall focus here on the maximum amplitude squared of the eigenfunctions on a single bond,

$$
\begin{aligned}
A_{i}(n, \mathbf{L} ; v) & :=\sup _{x \in\left[0, L_{i}\right]}\left\{\left|\psi_{i}^{(n)}(x)\right|^{2}\right\} \\
& =\left(A^{(n)} \sec k_{n} L_{i}\right)^{2} \\
& =\frac{2 \sec ^{2} k_{n} L_{i}}{\sum_{j=1}^{v} L_{j} \sec ^{2} k_{n} L_{j}} .
\end{aligned}
$$

We now state our main results.

Theorem 1. For any fixed $\bar{L}>0$

$$
\lim _{k \rightarrow \infty} \frac{1}{(\Delta L)^{v}} \operatorname{meas}\left\{\mathbf{L} \in[\bar{L}, \bar{L}+\Delta L]^{v}: \frac{1}{v} Z(k, \mathbf{L})<y\right\}=\frac{1}{\pi} \int_{-\infty}^{y} \frac{1}{1+x^{2}} \mathrm{~d} x,
$$

provided that $k \Delta L \rightarrow \infty$ as $k \rightarrow \infty$.

We emphasise that in theorem 1 we do not require that $\Delta L \rightarrow 0$. In some later results we shall make this stipulation.

Theorem 2. Suppose that the components of $\mathbf{L}$ are fixed and linearly independent over $\mathbb{Q}$. Then

$$
\lim _{K \rightarrow \infty} \frac{1}{K} \operatorname{meas}\left\{k \in[0, K]: \frac{1}{v} Z(k, \mathbf{L})<y\right\}=\frac{1}{\pi} \int_{-\infty}^{y} \frac{1}{1+x^{2}} \mathrm{~d} x .
$$

Theorems 1 and 2 demonstrate the equivalence of taking a $k$-average and a bond-length average at large $k$ for the distribution of values taken by the function $Z(k, \mathbf{L})$. Such a correspondence was noted in [2] for the spacing distribution of the eigenvalues of quantum graphs.

In [17] it was shown that the value distributions of the real and imaginary parts of the logarithm of the characteristic polynomial of a random unitary matrix drawn from the circular ensembles of random matrix theory tend independently to a Gaussian distribution in the limit as the matrix size tends to infinity, subject to appropriate normalisation. The distribution that appears 
in theorems 10 and 2 is known as the Cauchy distribution. It is related to the Gaussian distribution by the fact that both are examples of a larger class of distributions known as stable distributions. Such distributions share the property that the sum of two random variables from a stable distribution is distributed like a random variable from the same distribution. Theorem 1 is a consequence of this fact. We also note that the density in theorems 1 and 2 is independent of $v$ when $Z(k, \mathbf{L})$ is normalised as indicated.

We next consider the distribution of values taken by $Z^{\prime}(k)$ when $k=k_{n}$, $n=1,2, \ldots$.

Theorem 3. Let the components of $\mathbf{L}$ be linearly independent over $\mathbb{Q}$. Then there exists a probability density $P_{v}(y)$, depending on $\mathbf{L}$, such that

$$
\lim _{N \rightarrow \infty} \frac{1}{N} \#\left\{n \in\{1, \ldots, N\}: \frac{1}{v^{2}} Z^{\prime}\left(k_{n}, \mathbf{L}\right)<R\right\}=\int_{-\infty}^{R} P_{v}(y) \mathrm{d} y,
$$

with $P_{v}(y)=0$ for $y<0$.

Theorem 4. For each $v$ let the bond lengths $L_{j}, j=1, \ldots, v$ lie in the range $[\bar{L}, \bar{L}+\Delta L]$ and be linearly independent over $\mathbb{Q}$. If $v \Delta L \rightarrow 0$ as $v \rightarrow \infty$ then for any $R \in \mathbb{R}$,

$$
\int_{-\infty}^{R} P_{v}(y) \mathrm{d} y \rightarrow \int_{-\infty}^{R} P(y) \mathrm{d} y
$$

as $v \rightarrow \infty$. The limiting density is given by the continuous function

$$
P(y)= \begin{cases}\frac{\sqrt{L}}{4 \pi y^{3 / 2}} \int_{-\infty}^{\infty} \exp \left(-\frac{\xi^{2}}{4}-\frac{\bar{L} m(\xi)^{2}}{4 y}\right) m(\xi) \mathrm{d} \xi, & y>0 \\ 0, & y \leq 0,\end{cases}
$$

where

$$
m(\xi):=\frac{2}{\sqrt{\pi}} \mathrm{e}^{-\xi^{2} / 4}+\xi \operatorname{erf}(\xi / 2) .
$$

By comparison, the value distribution for the logarithm of the derivative of the characteristic polynomial of a matrix drawn from the CUE of random matrix theory, evaluated at an eigenvalue in the limit as matrix size tends to infinity, is Gaussian [16].

The following results refer to the value distribution of $A_{i}(n, \mathbf{L} ; v)$.

Theorem 5. Assume the conditions of theorem 3 are satisfied. Then there exists a probability density $Q_{v}(\eta)$ such that

$$
\lim _{N \rightarrow \infty} \frac{1}{N} \#\left\{n \in\{1, \ldots, N\}: v^{2} A_{i}(n, \mathbf{L} ; v)<R\right\}=\int_{0}^{R} Q_{v}(\eta) \mathrm{d} \eta
$$

where the density $Q_{v}(\eta)$ is independent of the choice of bond $i$ but depends on L. 
Theorem 6. Assume the conditions of theorem 4 are satisfied. Then for each $R>0$

$$
\int_{0}^{R} Q_{v}(\eta) \mathrm{d} \eta \rightarrow \int_{0}^{R} Q(\eta) \mathrm{d} \eta
$$

as $v \rightarrow \infty$. The limiting density is given by the function

$$
Q(\eta)=\frac{1}{2 \pi^{3 / 2} \eta} \mathfrak{I m} \int_{-\infty}^{\infty} \exp \left(-\frac{\xi^{2}}{4}-\frac{\bar{L} \eta m(\xi)^{2}}{8}\right) \operatorname{erfc}\left(\frac{\sqrt{\bar{L} \eta} m(\xi)}{2 \mathrm{i} \sqrt{2}}\right) \mathrm{d} \xi
$$

which is continuous on $(0, \infty)$. Here $m(\xi)$ is as in theorem $4 Q(\eta)$ has asymptotic expansion

$$
Q(\eta)=\frac{\sqrt{2}}{\sqrt{\bar{L}} \pi^{2} \eta^{3 / 2}} \int_{-\infty}^{\infty} \frac{\mathrm{e}^{-\xi^{2} / 4}}{m(\xi)} \mathrm{d} \xi+\mathrm{O}\left(\eta^{-5 / 2}\right)
$$

as $\eta \rightarrow \infty$.

The proofs of theorems 3 , 6 rely on an equidistribution result of Barra and Gaspard [2]. We review this work in section 3]

The limit $v \rightarrow \infty$ is analogous to the semiclassical limit $\hbar \rightarrow 0$ 20. We note that theorem [ 6 describes the wave functions on a vanishingly small fraction of the graph. It thus goes beyond the information provided by the Schnirelman theorem. It instead corresponds to the Gaussian value distribution for the wave functions of classically chaotic systems implied by the random wave model [6].

The value distribution of the eigenvector components of asymptotically large random matrices are particular cases of the $\chi_{\beta}^{2}$ density

$$
P_{\chi_{\beta}^{2}}(\eta)=\left(\frac{\beta}{2}\right)^{\beta / 2} \eta^{\beta / 2-1} \Gamma^{-1}\left(\frac{\beta}{2}\right) \mathrm{e}^{-\beta \eta / 2},
$$

where the parameter $\beta$ takes the values 1, 2 and 4 in, respectively, the orthogonal, unitary and symplectic ensembles (see for example [15]). When $\beta=1$ the density is called the Porter-Thomas density. It is characterised by $\mathrm{O}\left(\eta^{-1 / 2}\right)$ behaviour as $\eta \rightarrow 0$ and $\mathrm{O}\left(\eta^{-1 / 2} \mathrm{e}^{-\eta / 2}\right)$ as $\eta \rightarrow \infty$. The limiting distribution we find in theorem [6] completely determines the value distribution of the star graph eigenfunctions (see the appendix) and has a significantly different shape (c.f. equation (4) and figure 7 below). Other quantum systems for which the value distribution of the eigenfunctions has a non-random-matrix limit are the Cat Maps [22].

In [3] a correspondence was noted between the two-point spectral correlation functions for star graphs and a class of systems known as Šeba billiards. The original Šeba billiard 28] was a rectangular quantum billiard perturbed by a point singularity. More generally, we describe any integrable system perturbed in such a way as belonging to the same class [29. We conjecture that the results derived in the present work will also apply to systems in the Šeba class.

The remainder of this paper is structured as follows. In section 2 we prove theorems 10 and 2. In section 3 we treat the finite $v$ cases, theorem 3 and 5 In sections 4 and 5 we prove, respectively, theorems 4 and 6 developing the necessary machinery in section 4 Section [6] is devoted to numerical computations that illustrate our results. We develop more fully the connections between the present work and Šeba billiards in section 7 


\section{The value distribution of $Z(\mathbf{L}, k)$}

Lemma 1. Let $\bar{L}>0$ and $\zeta$ be real constants, then

$$
\lim _{k \rightarrow \infty} \frac{1}{\Delta L} \int_{\bar{L}}^{\bar{L}+\Delta L} \exp (\mathrm{i} \zeta \tan k L) \mathrm{d} L=\mathrm{e}^{-|\zeta|}
$$

uniformly for $k \Delta L \rightarrow \infty$ as $k \rightarrow \infty$.

Proof. By the periodicity of the integrand we may shift the range of integration by multiples of $\pi / k$ so that without loss of generality we may take $\bar{L}$ in the range $0 \leq \bar{L} \leq \pi / k$. We write $\Delta L=\pi(n+p) / k$ where $n \in \mathbb{Z}$ and $0 \leq p<1$. Then by the periodicity of the integrand,

$$
\begin{aligned}
\int_{\bar{L}}^{\bar{L}+\pi n / k+\pi p / k} \exp (\mathrm{i} \zeta \tan k L) \mathrm{d} L \\
=\int_{\bar{L}}^{\bar{L}+p \pi / k} \exp (\mathrm{i} \zeta \tan k L) \mathrm{d} L+n \int_{0}^{\pi / k} \exp (\mathrm{i} \zeta \tan k L) \mathrm{d} L \\
=\int_{\bar{L}}^{\bar{L}+p \pi / k} \exp (\mathrm{i} \zeta \tan k L) \mathrm{d} L+\frac{n}{k} \int_{-\infty}^{\infty} \frac{\mathrm{e}^{\mathrm{i} \zeta z}}{1+z^{2}} \mathrm{~d} z
\end{aligned}
$$

where the substitution $z=\tan k L$ has been made. We note now that $n / k \Delta L \rightarrow$ $\pi^{-1}$ as $k \rightarrow \infty$ and

$$
\left|\frac{1}{\Delta L} \int_{\bar{L}}^{\bar{L}+p \pi / k} \exp (\mathrm{i} \zeta \tan k L) \mathrm{d} L\right| \leq \frac{\pi}{k \Delta L} \rightarrow 0 \quad \text { as } k \rightarrow \infty .
$$

A simple application of Cauchy's residue theorem allows us to evaluate the integral

$$
\int_{-\infty}^{\infty} \frac{\mathrm{e}^{\mathrm{i} \zeta z}}{1+z^{2}} \mathrm{~d} z=\pi \mathrm{e}^{-|\zeta|}
$$

Proof of theorem [1] We use here the characteristic function. With bond lengths chosen from a uniform distribution,

$$
\begin{aligned}
\mathbb{E}_{\mathrm{L}}(\exp (\mathrm{i} \zeta Z(k, \mathbf{L}))) & =\frac{1}{(\Delta L)^{v}} \int_{\bar{L}}^{\bar{L}+\Delta L} \cdots \int_{\bar{L}}^{\bar{L}+\Delta L} \exp \left(\mathrm{i} \zeta \sum_{j=1}^{v} \tan k L_{j}\right) \mathrm{d} L_{1} \cdots \mathrm{d} L_{v} \\
& =\left(\frac{1}{\Delta L} \int_{\bar{L}}^{\bar{L}+\Delta L} \exp (\mathrm{i} \zeta \tan k L) \mathrm{d} L\right)^{v}
\end{aligned}
$$

The subscript $\mathrm{L}$ indicates that the expectation is with respect to an average over bond lengths. Since the map $t \mapsto t^{v}$ is continuous, lemma 1 together with (5) allows us to deduce that

$$
\lim _{k \rightarrow \infty} \mathbb{E}_{\mathrm{L}}(\exp (\mathrm{i} \zeta Z(k, \mathbf{L})))=\mathrm{e}^{-v|\zeta|} .
$$


The limiting density corresponding to the characteristic function on the right hand side is given by

$$
P_{Z}(x)=\frac{1}{2 \pi} \int_{-\infty}^{\infty} \exp (-\mathrm{i} \zeta x-v|\zeta|) \mathrm{d} \zeta=\frac{1}{\pi} \frac{v}{v^{2}+x^{2}} .
$$

The theorem follows now from the classical continuity theorem for characteristic functions (14] chapter XV).

The proof of theorem 2uses Weyl's Equidistribution theorem. This celebrated result 31 has numerous applications in analysis and number theory. We state here the form most convenient for application to our current work. Let $\mathbb{T}^{v}$ be the $v$-dimensional torus, with sides of length $\pi$.

Theorem 7. Let $f \in C\left(\mathbb{T}^{v}\right)$, and let the components of $\mathbf{L}$ be linearly independent over $\mathbb{Q}$. Then

$$
\lim _{K \rightarrow \infty} \frac{1}{K} \int_{0}^{K} f\left(L_{1} k, \ldots, L_{v} k\right) \mathrm{d} k=\frac{1}{\pi^{v}} \int_{\mathbb{T}^{v}} f(\mathbf{x}) \mathrm{d} \mathbf{x}
$$

where $\mathrm{d} \mathbf{x}=\mathrm{d} x_{1} \cdots \mathrm{d} x_{v}$ denotes Lebesgue measure.

We shall use Weyl's theorem as our main tool to relate $k$-averages to bond length averages. It is for this reason that it is crucial that the bond lengths are incommensurate.

We remark that theorem 7 can also apply to more general functions such as piecewise continuous functions through an argument similar to the one in the following lemma.

Lemma 2. Theorem 7 can also be applied to the function

$$
f(\mathbf{x}):=\exp \left(\mathrm{i} \zeta \sum_{j=1}^{v} \tan x_{j}\right) .
$$

Proof. We treat the real and imaginary parts of $f$ separately. The functions

$$
\begin{aligned}
& f_{1}(\mathbf{x}):=\cos \left(\zeta \sum_{j=1}^{v} \tan x_{j}\right) \\
& f_{2}(\mathbf{x}):=\sin \left(\zeta \sum_{j=1}^{v} \tan x_{j}\right)
\end{aligned}
$$

are smooth everywhere apart from at an essential singularity when $x_{i}=\pi / 2$ for some $i$, which we tame in the following way. Let $\epsilon>0$. We can construct functions $\phi$ and $\psi$ satisfying the conditions of theorem 7 such that

$$
\begin{aligned}
& \psi(\mathbf{x})=-1 \text { if }\left|x_{i}-\pi / 2\right|<\pi \epsilon^{1 / v} / 8 \text { for some } i=1, \ldots, v, \\
& \phi(\mathbf{x})=1 \text { if }\left|x_{i}-\pi / 2\right|<\pi \epsilon^{1 / v} / 8 \text { for some } i=1, \ldots, v, \\
& \psi(\mathbf{x})=\phi(\mathbf{x})=f_{1}(\mathbf{x}) \begin{array}{l}
\text { if } \pi \epsilon^{1 / v} / 4<\left|x_{i}-\pi / 2\right|<\pi / 2 \text { for some } \\
i=1, \ldots, v
\end{array} \\
&-1 \leq \psi(\mathbf{x}) \leq f_{1}(\mathbf{x}) \leq \phi(\mathbf{x}) \leq 1 \text { for all } \mathbf{x} \in \mathbb{T}^{v} .
\end{aligned}
$$

This implies

$$
\frac{1}{\pi^{v}} \int_{\mathbb{T}^{v}}(\phi(\mathbf{x})-\psi(\mathbf{x})) \mathrm{d} \mathbf{x} \leq \frac{1}{\pi^{v}}\left(2 \frac{\pi}{2} \epsilon^{1 / v}\right)^{v}=\epsilon .
$$


From (7) and theorem 7

$$
\begin{aligned}
\frac{1}{\pi^{v}} \int_{\mathbb{T}^{v}} \psi(\mathbf{x}) \mathrm{d} \mathbf{x} \leq \liminf _{K \rightarrow \infty} & \frac{1}{K} \int_{0}^{K} f_{1}(k \mathbf{L}) \mathrm{d} k \\
& \leq \limsup _{K \rightarrow \infty} \frac{1}{K} \int_{0}^{K} f_{1}(k \mathbf{L}) \mathrm{d} k \leq \frac{1}{\pi^{v}} \int_{\mathbb{T} v} \phi(\mathbf{x}) \mathrm{d} \mathbf{x} .
\end{aligned}
$$

The ends of this inequality differ by $\epsilon$ which can be made arbitrarily small, so we see that $\lim _{K \rightarrow \infty} K^{-1} \int_{0}^{K} f_{1}(k \mathbf{L}) \mathrm{d} k$ exists and is equal to

$$
\frac{1}{\pi^{v}} \int_{\mathbb{T}^{v}} f_{1}(\mathbf{x}) \mathrm{d} \mathbf{x} .
$$

The extension to $f_{2}$ and hence $f$ is obvious.

Proof of theorem 2 We begin in the same way as in the proof of theorem 1 In this case $k$ is chosen uniformly from the interval $[0, K]$ with $K>0$, so that the characteristic function with respect to this uniform distribution is

$$
\mathbb{E}_{K}(\exp (\mathrm{i} \zeta Z(k, \mathbf{L})))=\frac{1}{K} \int_{0}^{K} \exp \left(\mathrm{i} \zeta \sum_{j=1}^{v} \tan k L_{j}\right) \mathrm{d} k .
$$

By lemma 2 we can write this integral as an average over the torus as $K \rightarrow \infty$ :

$$
\begin{aligned}
\lim _{K \rightarrow \infty} \mathbb{E}_{K}(\exp (\mathrm{i} \zeta Z(k, \mathbf{L}))) & =\frac{1}{\pi^{v}} \int_{\mathbb{T}^{v}} \exp \left(\mathrm{i} \zeta \sum_{j=1}^{v} \tan x_{j}\right) \mathrm{d} \mathbf{x} \\
& =\left(\frac{1}{\pi} \int_{0}^{\pi} \exp (\mathrm{i} \zeta \tan x) \mathrm{d} x\right)^{v} .
\end{aligned}
$$

Following the substitution $z=\tan x$ in this final integral, we have

$$
\lim _{K \rightarrow \infty} \mathbb{E}_{K}\left(\exp (\mathrm{i} \zeta Z(k, \mathbf{L}))=\mathrm{e}^{-v|\zeta|}\right.
$$

and the theorem follows from the same arguments used in the end of the proof of theorem 1 .

\section{An equidistribution theorem}

Barra and Gaspard [2] observed that the condition for $k$ to be an eigenvalue of a graph can be written in the form

$$
G(k \mathbf{L})=0,
$$

where $G$ is a function that is periodic in each variable. For star graphs $G$ is defined on $\mathbb{T}^{v}$ by

$$
G(\mathbf{x})=\tan x_{1}+\cdots+\tan x_{v} .
$$

The equation

$$
G(\mathbf{x})=0
$$


defines a surface $\Sigma$ embedded in $\mathbb{T}^{v}$. A flow $\phi^{k}, k \in \mathbb{R}$ can be defined on $\mathbb{T}^{v}$ by

$$
\phi^{k}\left(\mathbf{x}_{0}\right)=\mathbf{x}_{0}+k \mathbf{L} \quad(\bmod \pi) .
$$

Since $k=0$ is an eigenvalue for star graphs with Neumann boundary conditions considered here, we take $\mathbf{x}_{0}=0$ in this case.

At each value $k=k_{n}$ we have an intersection of this flow with the surface $\Sigma$. We note that the angle between the normal to the surface $\Sigma$ and the flow $\phi^{k}$ is given by

$$
\cos \theta=\frac{|\mathbf{L} \cdot \nabla G|}{\|\mathbf{L}\|\|\nabla G\|} .
$$

For star graphs,

$$
\nabla G(\mathbf{x})=\left(\sec ^{2} x_{1}, \ldots, \sec ^{2} x_{v}\right) .
$$

Hence there exists a constant $c_{1}>0$ such that $\cos \theta>c_{1}$. This means that the angle between the flow and the surface $\Sigma$ is uniformly bounded away from 0 . We can therefore parameterise $\Sigma$ locally by $v-1$ real variables $\boldsymbol{\xi}=\left(\xi_{1}, \ldots, \xi_{v-1}\right)$ so that for $\mathbf{x} \in \Sigma$,

$$
x_{i}=s_{i}(\boldsymbol{\xi})
$$

and $G\left(s_{1}(\boldsymbol{\xi}), \ldots, s_{v}(\boldsymbol{\xi})\right)=0$.

The central result of Barra and Gaspard is the existence of an invariant measure on the surface $\Sigma$.

Theorem 8. Let $f$ be a piecewise continuous function $\Sigma \rightarrow \mathbb{R}$. Then

$$
\lim _{N \rightarrow \infty} \frac{1}{N} \sum_{n=1}^{N} f\left(k_{n} \mathbf{L}\right)=\int_{\Sigma} f(\boldsymbol{\xi}) \mathrm{d} \nu(\boldsymbol{\xi})
$$

where the measure $\nu$ is given by

$$
\mathrm{d} \nu(\boldsymbol{\xi})=\frac{J(\boldsymbol{\xi}) \mathrm{d} \boldsymbol{\xi}}{\int_{\Sigma} J(\boldsymbol{\xi}) \mathrm{d} \boldsymbol{\xi}}
$$

and $\mathrm{d} \boldsymbol{\xi}=\mathrm{d} \xi_{1} \cdots \mathrm{d} \xi_{v-1}$ is Lebesgue measure. $J$ is the Jacobian determinant

$$
J(\boldsymbol{\xi})=\left|\begin{array}{ccc}
L_{1} & \cdots & L_{v} \\
\frac{\partial s_{1}}{\partial \xi_{1}} & \cdots & \frac{\partial s_{v}}{\partial \xi_{1}} \\
\vdots & \ddots & \vdots \\
\frac{\partial s_{1}}{\partial \xi_{v-1}} & \cdots & \frac{\partial s_{v}}{\partial \xi_{v-1}}
\end{array}\right|
$$

For completeness, we sketch a proof of theorem 8 for star graphs with $v$ bonds. Proof. Let $\tilde{f}: \mathbb{T}^{v} \rightarrow \mathbb{R}$ be an extension of $f$ to $\mathbb{T}^{v}$, so that $\left.\tilde{f}\right|_{\Sigma}=f$, i.e.

$$
\tilde{f}(\mathbf{x})=f(\mathbf{x}) \quad \text { for all } \mathbf{x} \in \Sigma .
$$

We let $\tilde{f}$ be constructed in such a way that for all $\boldsymbol{\xi} \in \Sigma, \tilde{f}\left(\phi^{k}(\boldsymbol{\xi})\right)$ is a differentiable function of $k$ with compact support in some neighbourhood of $k=0$.

Let $\epsilon>0$. We construct the set $\Sigma_{\epsilon, \mathbf{L}}$ which is a thickening of $\Sigma$ in the direction of the flow $\phi^{k}$,

$$
\Sigma_{\epsilon, \mathbf{L}}:=\left\{\mathbf{x} \in \mathbb{T}^{v}: \exists \boldsymbol{\xi} \in \Sigma, k \in[-\epsilon, \epsilon]: \mathbf{x}=\phi^{k}(\boldsymbol{\xi})\right\} \subseteq \mathbb{T}^{v} .
$$


We define $\mathbb{1}_{A}$, the indicator function of a set $A$, by

$$
\mathbb{1}_{A}(x):=\left\{\begin{array}{l}
1, x \in A \\
0, x \notin A
\end{array}\right.
$$

The indicator function $\mathbb{1}_{\Sigma_{\epsilon, \mathbf{L}}}(\mathbf{x})$ is piecewise constant.

By the differentiability properties of $\tilde{f}$ we can write for every $\mathbf{x} \in \Sigma$

$$
f(\mathbf{x})=\tilde{f}(\mathbf{x})=\frac{1}{2 \epsilon} \int_{-\epsilon}^{\epsilon} \tilde{f}(k \mathbf{L}+\mathbf{x}) \mathrm{d} k+\mathrm{O}(\epsilon)
$$

as $\epsilon \rightarrow 0$. The implied constant does not depend on $\mathbf{x}$. Setting $\mathbf{x}=k_{n} \mathbf{L}$ gives

$$
\frac{1}{N} \sum_{n=1}^{N} f\left(k_{n} \mathbf{L}\right)=\frac{1}{2 \epsilon N} \sum_{n=1}^{N} \int_{-\epsilon}^{\epsilon} \tilde{f}\left(\left(k+k_{n}\right) \mathbf{L}\right) \mathrm{d} k+\mathrm{O}(\epsilon) .
$$

Let the mean density of zeros of $Z(k, \mathbf{L})$ be $\bar{d}$ :

$$
\bar{d}:=\lim _{K \rightarrow \infty} \frac{\#\left\{n: k_{n} \leq K\right\}}{K} .
$$

Then

$$
\begin{aligned}
\lim _{N \rightarrow \infty} \frac{1}{N} \sum_{n=1}^{N} f\left(k_{n} \mathbf{L}\right) & =\frac{1}{2 \epsilon \bar{d}} \lim _{K \rightarrow \infty} \frac{1}{K} \int_{0}^{K} \tilde{f}(k \mathbf{L}) \mathbb{1}_{\Sigma_{\epsilon, \mathbf{L}}}(k \mathbf{L}) \mathrm{d} k+\mathrm{O}(\epsilon) \\
& =\frac{1}{\pi^{v} \bar{d}} \int_{\mathbb{T}^{v}} \tilde{f}(\mathbf{x}) \mathbb{1}_{\Sigma_{\epsilon, \mathbf{L}}}(\mathbf{x}) \mathrm{d} \mathbf{x}+\mathrm{O}(\epsilon),
\end{aligned}
$$

applying theorem $\mathbf{7}$ to the piecewise continuous function $\tilde{f}(\mathbf{x}) \mathbb{1}_{\Sigma_{\epsilon, \mathrm{L}}}(\mathbf{x})$. Changing to the system of coordinates $(t, \boldsymbol{\xi})$ on $\mathbb{T}^{v}$ via the change of variables

$$
x_{i}=L_{i} t+s_{i}(\boldsymbol{\xi}),
$$

gives

$$
\lim _{N \rightarrow \infty} \frac{1}{N} \sum_{n=1}^{N} f\left(k_{n} \mathbf{L}\right)=\frac{1}{\pi^{v} \bar{d}} \int_{\Sigma} \frac{1}{2 \epsilon} \int_{-\epsilon}^{\epsilon} \tilde{f}(t, \boldsymbol{\xi}) J(\boldsymbol{\xi}) \mathrm{d} t \mathrm{~d} \boldsymbol{\xi}+\mathrm{O}(\epsilon) .
$$

Since this is true for all $\epsilon>0$, we deduce that

$$
\lim _{N \rightarrow \infty} \frac{1}{N} \sum_{n=1}^{N} f\left(k_{n} \mathbf{L}\right)=\frac{1}{\pi^{v} \bar{d}} \int_{\Sigma} f(\boldsymbol{\xi}) J(\boldsymbol{\xi}) \mathrm{d} \boldsymbol{\xi} .
$$

By setting $f=1$, we see that

$$
\bar{d}=\frac{1}{\pi^{v}} \int_{\Sigma} J(\boldsymbol{\xi}) \mathrm{d} \boldsymbol{\xi}
$$

to complete the proof. 
We note incidentally that $\bar{d}$ can be evaluated using spectral methods [20] to give

$$
\bar{d}=\frac{1}{\pi} \sum_{j=1}^{v} L_{j}=\frac{v \bar{L}}{\pi}+\mathrm{O}(v \Delta L) \quad \text { as } v \Delta L \rightarrow 0 .
$$

We observe that the right hand side of equation (9) can formally be written in the form

$$
\int_{\Sigma} f(\boldsymbol{\xi}) \mathrm{d} \nu(\boldsymbol{\xi})=\frac{1}{2 \pi^{v+1} \bar{d}} \int_{-\infty}^{\infty} \int_{0}^{\pi} \cdots \int_{0}^{\pi} f(\mathbf{x})[\mathbf{L} \cdot \nabla G(\mathbf{x})] \mathrm{e}^{\mathrm{i} \zeta G} \mathrm{~d} x_{1} \cdots \mathrm{d} x_{v} \mathrm{~d} \zeta
$$

where now $f$ is a function $\mathbb{T}^{v} \rightarrow \mathbb{R}$ of an appropriate class. This follows from writing $\int_{\Sigma} f(\boldsymbol{\xi}) \mathrm{d} \nu(\boldsymbol{\xi})$ in the equivalent form,

$$
\frac{1}{\pi^{v} \bar{d}} \int_{\Sigma} \int f(t, \boldsymbol{\xi})[\mathbf{L} \cdot \nabla G] \delta(G(t, \boldsymbol{\xi})) J(\boldsymbol{\xi}) \mathrm{d} t \mathrm{~d} \boldsymbol{\xi} .
$$

We then write the $\delta$-function as the limit of the sequence

$$
\delta_{m}(x):=\frac{1}{2 \pi} \int_{-m}^{m} \mathrm{e}^{\mathrm{i} \zeta x} \mathrm{~d} \zeta=\frac{\sin m x}{\pi x}
$$

as $m \rightarrow \infty$, and changing back to the usual Cartesian coordinates on $\mathbb{T}^{v}$. We need to show that $\delta_{m}$ is an appropriate $\delta$-sequence for the function $f$ that we consider. We will check this point directly in the calculations where the identity (15) is used. We shall also justify taking the $\zeta$-integral in (17) outside the integral over $\mathbb{T}^{v}$.

Proposition 1. For a star graph with $v$ bonds and the parameterisation

$$
\begin{array}{r}
s_{i}=\xi_{i} \quad i=1, \ldots, v-1 \\
s_{v}=-\tan ^{-1}\left(\tan \xi_{1}+\cdots+\tan \xi_{v-1}\right)
\end{array}
$$

$J(\boldsymbol{\xi})$ takes the following form

$$
J(\boldsymbol{\xi})=\frac{L_{1} \sec ^{2} \xi_{1}+\cdots+L_{v-1} \sec ^{2} \xi_{v-1}}{1+\left(\tan \xi_{1}+\cdots+\tan \xi_{v-1}\right)^{2}}+L_{v}
$$

Proof. Differentiating gives

$$
\frac{\partial s_{i}}{\partial \xi_{j}}=\delta_{i j}
$$

for $i<v$ and

$$
\frac{\partial s_{v}}{\partial \xi_{j}}=\frac{-\sec ^{2} \xi_{j}}{1+\left(\tan \xi_{1}+\cdots+\tan \xi_{v-1}\right)^{2}} .
$$

For ease of notation we write $D:=1+\left(\tan \xi_{1}+\cdots+\tan \xi_{v-1}\right)^{2}$. Thus we have

$$
J(\boldsymbol{\xi})=\left|\begin{array}{ccccc}
L_{1} & L_{2} & \cdots & L_{v-1} & L_{v} \\
1 & 0 & \cdots & 0 & \frac{-\sec ^{2} \xi_{1}}{D} \\
0 & 1 & \cdots & 0 & \frac{-\sec ^{2} \xi_{2}}{D} \\
\vdots & \vdots & \ddots & \vdots & \vdots \\
0 & 0 & \cdots & 1 & \frac{-\sec ^{2} \xi_{v-1}}{D}
\end{array}\right| .
$$


To complete the proof we employ the identity

$$
\left|\begin{array}{ccccc}
\alpha_{1} & \alpha_{2} & \cdots & \alpha_{n-1} & \alpha_{n} \\
1 & 0 & \cdots & 0 & \beta_{1} \\
0 & 1 & \cdots & 0 & \beta_{2} \\
\vdots & \vdots & \ddots & \vdots & \vdots \\
0 & 0 & \cdots & 1 & \beta_{n-1}
\end{array}\right|=(-1)^{n}\left(-\alpha_{n}+\sum_{k=1}^{n-1} \alpha_{k} \beta_{k}\right)
$$

which may be readily checked by induction.

We note in passing that the explicit form of $J(\boldsymbol{\xi})$ given above together with theorem 8 provides a convenient representation for use numerical studies of eigenvalues and eigenfunctions because the zeros of $Z(k, \mathbf{L})$ do not need to be computed explicitly.

Proof of theorem [3. We take as the function $f$ in theorem 8

$$
f(\mathbf{x})=\mathbb{1}_{(-\infty, R]}\left(\frac{1}{v^{2}} \sum_{j=1}^{v} L_{j} \sec ^{2} x_{j}\right)
$$

Then we define $P_{v}(y)$ by

$$
\begin{array}{r}
\int_{-\infty}^{R} P_{v}(y) \mathrm{d} y=\frac{1}{\pi^{v} \bar{d}} \int_{0}^{\pi} \cdots \int_{0}^{\pi} f\left(\xi_{1}, \ldots, \xi_{v-1},-\tan ^{-1}\left(\tan \xi_{1}+\cdots+\tan \xi_{v-1}\right)\right) \\
\times J(\boldsymbol{\xi}) \mathrm{d} \xi_{1} \cdots \mathrm{d} \xi_{v-1},
\end{array}
$$

where $J(\boldsymbol{\xi})$ is defined by (18). Since $\sec ^{2} x>0$ for all $x \in \mathbb{R}$, it follows that $P_{v}(y)=0$ for $y<0$.

Proof of theorem [ In this case, we take as the function $f$ in theorem 8

$$
f(\mathbf{x})=\mathbb{1}_{[0, R]}\left(\frac{2 v^{2} \sec ^{2} x_{i}}{\sum_{j} L_{j} \sec ^{2} x_{j}}\right) .
$$

We take as the parameterisation of $\Sigma$

$$
\begin{aligned}
& s_{j}=\xi_{j}, \quad 1 \leq j<i \\
& s_{i}=-\tan ^{-1}\left(\tan \xi_{1}+\cdots+\tan \xi_{v-1}\right) \\
& s_{j}=\xi_{j-1}, \quad i<j \leq v .
\end{aligned}
$$

This does not change the form of $J(\boldsymbol{\xi})$ from that in (18), but introduces extra symmetry in $f$. Then define, as before,

$$
\int_{0}^{R} P_{v}(\eta) \mathrm{d} \eta=\frac{1}{\pi^{v} \bar{d}} \int_{0}^{\pi} \cdots \int_{0}^{\pi} f\left(s_{1}(\boldsymbol{\xi}), \ldots, s_{v}(\boldsymbol{\xi})\right) J(\boldsymbol{\xi}) \mathrm{d} \xi_{1} \cdots \mathrm{d} \xi_{v-1} .
$$

Since $f(\mathbf{s}(\boldsymbol{\xi}))$ is symmetric in $\xi_{1}, \ldots, \xi_{v-1}$ we see that $P_{v}(\eta)$ is independent of the choice of bond $i$. 


\section{Value distribution of $Z^{\prime}\left(k_{n}\right)$ in the limit $v \rightarrow \infty$}

We take as the function $f$ in (15) the characteristic function for the value distribution of the derivative of $Z(k, \mathbf{L})$,

$$
Z^{\prime}(k, \mathbf{L})=\sum_{j=1}^{v} L_{j} \sec ^{2} k L_{j} .
$$

Since we are stipulating that $v \Delta L \rightarrow 0$ as $v \rightarrow \infty$, we replace $L_{j}$ by $\bar{L}$ where it does not multiply $k$ in (19) and take as our $f$

$$
f(\mathbf{x})=\exp \left(-\frac{\mathrm{i} \beta \bar{L}}{v^{2}} \sum_{j} \sec ^{2} x_{j}\right) .
$$

Let the quantity in which we are interested be denoted $E_{v}(\beta)$. Then

$$
\begin{aligned}
E_{v}(\beta):= & \lim _{N \rightarrow \infty} \frac{1}{N} \sum_{n=1}^{N} \exp \left(-\frac{\mathrm{i} \beta \bar{L}}{v^{2}} \sum_{j} \sec ^{2} k_{n} L_{j}\right) \\
= & \frac{\bar{L}}{2 \pi \bar{d} v} \int_{-\infty}^{\infty} \frac{1}{\pi^{v}} \int_{0}^{\pi} \cdots \int_{0}^{\pi}\left(\sum_{j} \sec ^{2} x_{j}\right) \\
& \times \prod_{j=1}^{v} \exp \left(-\frac{\mathrm{i} \beta \bar{L}}{v^{2}} \sec ^{2} x_{j}+\frac{\mathrm{i} \zeta}{v} \sum_{j} \tan x_{j}\right) \mathrm{d} \mathbf{x} \mathrm{d} \zeta .
\end{aligned}
$$

We have made the re-scaling $\zeta \mapsto \zeta / v$. This is a natural normalisation since $Z(k, \mathbf{L})$ is a sum of $v$ terms.

We exploit the symmetry in the integral in (20) to write

$$
E_{v}(\beta)=\frac{1}{2 v} \int_{-\infty}^{\infty} I_{1}(\beta, \zeta)\left(I_{2}(\beta, \zeta)\right)^{v-1} \mathrm{~d} \zeta
$$

where we have replaced $\bar{d}$ by $\bar{L} v / \pi$ and defined the integrals

$$
I_{1}(\beta, \zeta):=\frac{1}{\pi} \int_{0}^{\pi} \sec ^{2} x \exp \left(-\frac{\mathrm{i} \beta \bar{L}}{v^{2}} \sec ^{2} x+\frac{\mathrm{i} \zeta}{v} \tan x\right) \mathrm{d} x
$$

and

$$
I_{2}(\beta, \zeta):=\frac{1}{\pi} \int_{0}^{\pi} \exp \left(-\frac{\mathrm{i} \beta \bar{L}}{v^{2}} \sec ^{2} x+\frac{\mathrm{i} \zeta}{v} \tan x\right) \mathrm{d} x .
$$

We note that $I_{2}$ is uniformly convergent in $\zeta$ but that $I_{1}$ is not. 
4.1. The integrals $I_{1}$ and $I_{2}$. The substitution $z=\tan x$ gives

$$
\begin{aligned}
I_{1}(\beta, \zeta) & =\frac{1}{\pi} \int_{-\infty}^{\infty} \exp \left(-\frac{\mathrm{i} \beta \bar{L}}{v^{2}}\left(1+z^{2}\right)+\frac{\mathrm{i} \zeta z}{v}\right) \mathrm{d} z \\
& =\frac{v}{\sqrt{\pi}} \frac{1}{\sqrt{\mathrm{i} \beta \bar{L}}} \exp \left(-\frac{\zeta^{2}}{4 \mathrm{i} \beta \bar{L}}-\frac{\mathrm{i} \beta \bar{L}}{v^{2}}\right)
\end{aligned}
$$

quoting a standard integral.

That $\delta_{m}$ defined in (17) is a $\delta$-sequence for the function $\exp \left(\mathrm{i} \alpha z^{2}\right)$ follows from the equality

$$
\frac{1}{2 \pi} \int_{-\infty}^{\infty} \int_{-\infty}^{\infty} \mathrm{e}^{\mathrm{i} \alpha z^{2}+\mathrm{i} \zeta(z-w)} \mathrm{d} z \mathrm{~d} \zeta=\exp \left(\mathrm{i} \alpha w^{2}\right)
$$

which can be checked by direct evaluation of the integrals. This and uniform convergence of $I_{2}$ in $\zeta$ justifies the operations that lead to identity (15).

We can treat $I_{2}$ in a similar manner to that in which we treated $I_{1}$.

$$
I_{2}(\beta, \zeta)=\frac{1}{\pi} \int_{-\infty}^{\infty} \exp \left(-\frac{\mathrm{i} \beta \bar{L}}{v^{2}}\left(1+z^{2}\right)+\frac{\mathrm{i} \zeta z}{v}\right) \frac{\mathrm{d} z}{1+z^{2}} .
$$

We first write

$$
\frac{1}{1+z^{2}}=\frac{1}{2 \mathrm{i}}\left(\frac{1}{z-\mathrm{i}}-\frac{1}{z+\mathrm{i}}\right)
$$

so that $I_{2}$ can be decomposed into a difference of two similar integrals

$$
I_{2}(\beta, \zeta)=: I_{2}^{-}(\beta, \zeta)-I_{2}^{+}(\beta, \zeta)
$$

Observing that

$$
\exp \left(-\frac{\mathrm{i} \beta \bar{L}}{v^{2}}\left(1+z^{2}\right)+\frac{\mathrm{i} \zeta z}{v}\right)=\exp \left(-\frac{\mathrm{i} \beta \bar{L}}{v^{2}}-\frac{\zeta^{2}}{4 \mathrm{i} \beta \bar{L}}-\frac{\mathrm{i} \beta \bar{L}}{v^{2}}\left(z+\frac{\zeta v}{2 \beta \bar{L}}\right)^{2}\right),
$$

we can write

$$
\begin{aligned}
I_{2}^{-}(\beta, \zeta) & :=\frac{1}{2 \pi \mathrm{i}} \int_{-\infty}^{\infty} \exp \left(-\frac{\mathrm{i} \beta \bar{L}}{v^{2}}\left(1+z^{2}\right)+\frac{\mathrm{i} \zeta z}{v}\right) \frac{\mathrm{d} z}{z-i} \\
& =\frac{1}{2 \pi \mathrm{i}} \exp \left(-\frac{\mathrm{i} \beta \bar{L}}{v^{2}}+\frac{\mathrm{i} \zeta^{2}}{4 \beta \bar{L}}\right) \int_{-\infty}^{\infty} \frac{\exp \left(-\mathrm{i} \beta \bar{L} y^{2} / v^{2}\right)}{y-\mathrm{i}-\zeta v / 2 \beta \bar{L}} \mathrm{~d} y
\end{aligned}
$$

via $y=z+\zeta v / 2 \beta \bar{L}$. We make the change of variable

$$
r=\frac{\sqrt{\mathrm{i} \beta \bar{L}}}{v} y
$$

This is permitted, because it rotates the contour of integration into the second and fourth quadrants of the complex plane, where the analytic function $\mathrm{e}^{-\mathrm{i} z^{2}}$ decays rapidly. 
In the case $\zeta v / 2 \beta \bar{L}>-1$ the new contour of integration avoids the pole at $y=\zeta v / 2 \beta \bar{L}+\mathrm{i}$ (figure 2) and Cauchy's Theorem yields

$$
I_{2}^{-}(\beta, \zeta)=\frac{1}{2 \pi \mathrm{i}} \exp \left(-\frac{\mathrm{i} \beta \bar{L}}{v^{2}}+\frac{\mathrm{i} \zeta^{2}}{4 \beta \bar{L}}\right) \int_{-\infty}^{\infty} \frac{\mathrm{e}^{-r^{2}}}{r-\frac{\sqrt{\mathrm{i} \beta \bar{L}}}{v}\left(\mathrm{i}+\frac{\zeta v}{2 \beta L}\right)} \mathrm{d} r .
$$

This integral is standard, and may be found in, for example, [1] (Equation 7.1.4):

$$
\frac{\mathrm{i}}{\pi} \int_{-\infty}^{\infty} \frac{\mathrm{e}^{-t^{2}}}{z-t} \mathrm{~d} t=\mathrm{e}^{-z^{2}} \operatorname{erfc}(-\mathrm{i} z), \quad \text { for } \mathfrak{I m} z>0 .
$$

The result we get is

$$
\begin{aligned}
I_{2}^{-}(\beta, \zeta)= & \frac{1}{2} \exp \left(-\frac{\mathrm{i} \beta \bar{L}}{v^{2}}+\frac{\mathrm{i} \zeta^{2}}{4 \beta \bar{L}}\right) \exp \left(-\frac{\mathrm{i} \beta \bar{L}}{v^{2}}\left(\mathrm{i}+\frac{\zeta v}{2 \beta \bar{L}}\right)^{2}\right) \\
& \times \operatorname{erfc}\left(\frac{\sqrt{\mathrm{i} \beta \bar{L}}}{v}\left(1-\frac{\mathrm{i} \zeta v}{2 \beta \bar{L}}\right)\right) \\
= & \frac{1}{2} \exp \left(\frac{\zeta}{v}\right) \operatorname{erfc}\left(\frac{\zeta}{2 \sqrt{\mathrm{i} \beta \bar{L}}}+\frac{\sqrt{\mathrm{i} \beta \bar{L}}}{v}\right) .
\end{aligned}
$$

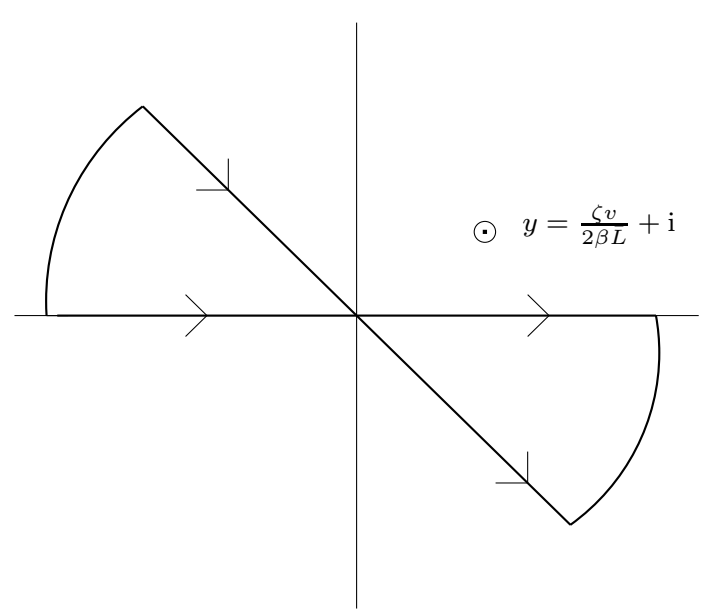

Fig. 2. Deforming the contour of integration avoiding pole

If $\zeta v / 2 \beta \bar{L}<-1$ then the contour encloses a pole (figure 3). In this case,

$$
\int_{-\infty}^{\infty} \frac{\exp \left(-\mathrm{i} \beta \bar{L} y^{2} / v^{2}\right)}{y-\mathrm{i}-\zeta v / 2 \beta \bar{L}} \mathrm{~d} y=2 \pi \mathrm{i} R+\int_{-\infty}^{\infty} \frac{\mathrm{e}^{-r^{2}}}{r-\frac{\sqrt{\mathrm{i} \beta \bar{L}}}{v}\left(\mathrm{i}+\frac{\zeta v}{2 \beta L}\right)} \mathrm{d} r
$$




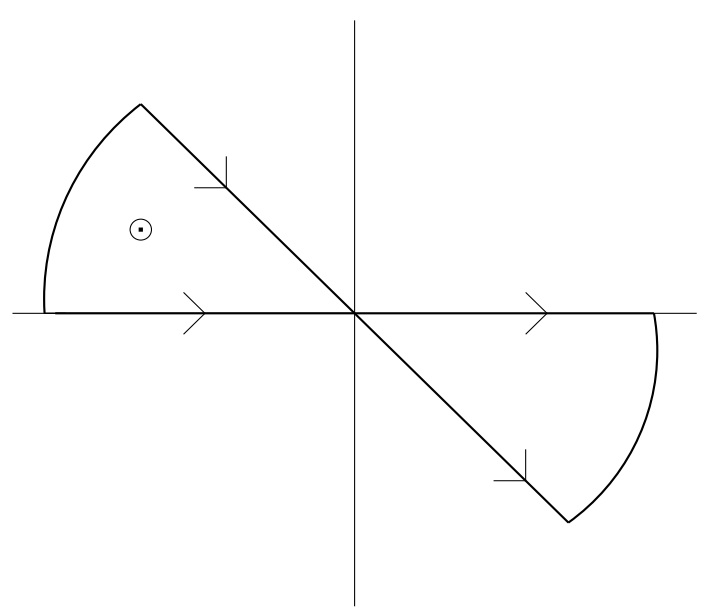

Fig. 3. Deforming the contour of integration enclosing pole

where $R$ is the residue at the pole

$$
\begin{aligned}
R & =\left.\exp \left(-\frac{\mathrm{i} \beta \bar{L} y^{2}}{v^{2}}\right)\right|_{y=\mathrm{i}+\zeta v / 2 \beta \bar{L}} \\
& =\exp \left(-\frac{\mathrm{i} \zeta^{2}}{4 \beta \bar{L}}+\frac{\mathrm{i} \beta \bar{L}}{v^{2}}+\frac{\zeta}{v}\right),
\end{aligned}
$$

so that we also get in this case

$$
I_{2}^{-}(\beta, \zeta)=\frac{1}{2} \exp \left(\frac{\zeta}{v}\right) \operatorname{erfc}\left(\frac{\zeta}{2 \sqrt{\mathrm{i} \beta \bar{L}}}+\frac{\sqrt{\mathrm{i} \beta \bar{L}}}{v}\right) .
$$

Treating $I_{2}^{+}$in a similar way, yields an expression for $I_{2}$,

$$
I_{2}(\beta, \zeta)=\frac{1}{2} \mathrm{e}^{\zeta / v} \operatorname{erfc}\left(\frac{\zeta}{2 \sqrt{\mathrm{i} \beta \bar{L}}}+\frac{\sqrt{\mathrm{i} \beta \bar{L}}}{v}\right)+\frac{1}{2} \mathrm{e}^{-\zeta / v} \operatorname{erfc}\left(\frac{-\zeta}{2 \sqrt{\mathrm{i} \beta \bar{L}}}+\frac{\sqrt{\mathrm{i} \beta \bar{L}}}{v}\right) .
$$

\subsection{Some estimates.}

Lemma 3. Let $-\sqrt{v} \leq \zeta \leq \sqrt{v}$, then

$$
\begin{aligned}
I_{1}(\beta, \zeta)\left(I_{2}(\beta, \zeta)\right)^{v-1}=\frac{v}{\sqrt{\pi}} & \frac{1}{\sqrt{\mathrm{i} \beta \bar{L}}} \exp \left(\frac{-\zeta^{2}}{4 \mathrm{i} \beta \bar{L}}\right) M(\beta, \zeta) \\
& \times\left(1+\mathrm{O}\left(v^{-1 / 2}\right)+\mathrm{O}\left(\zeta^{2} / v\right)\right)
\end{aligned}
$$


as $v \rightarrow \infty$, where

$$
M(\beta, \zeta):=\exp \left(-\frac{2 \sqrt{\mathrm{i} \beta \bar{L}}}{\sqrt{\pi}} \exp \left(\frac{-\zeta^{2}}{4 \mathrm{i} \beta \bar{L}}\right)-\zeta \operatorname{erf}\left(\frac{\zeta}{2 \sqrt{\mathrm{i} \beta \bar{L}}}\right)\right) .
$$

Proof. A key step will be to make a uniform expansion of $I_{2}(\beta, \zeta)$ as $v \rightarrow \infty$. By Taylor's theorem,

$$
\exp \left( \pm \frac{\zeta}{v}\right)=1 \pm \frac{\zeta}{v}+\mathrm{O}\left(\zeta^{2} v^{-2}\right) \quad \text { as } v \rightarrow \infty
$$

A second application of Taylor's theorem yields

$\operatorname{erfc}\left(\frac{ \pm \zeta}{2 \sqrt{\mathrm{i} \beta \bar{L}}}+\frac{\sqrt{\mathrm{i} \beta \bar{L}}}{v}\right)=\operatorname{erfc}\left(\frac{ \pm \zeta}{2 \sqrt{\mathrm{i} \beta \bar{L}}}\right)-\frac{2}{\sqrt{\pi}} \frac{\sqrt{\mathrm{i} \beta \bar{L}}}{v} \exp \left(-\frac{\zeta^{2}}{4 \mathrm{i} \beta \bar{L}}\right)+\frac{R_{1}(\zeta)}{v^{2}}$,

where the remainder term is

$$
R_{1}(\zeta)=-\left.\mathrm{i} \beta \bar{L} \int_{0}^{1} \frac{\mathrm{d}^{2}}{\mathrm{~d} z^{2}} \operatorname{erfc}(z)\right|_{z=\frac{ \pm \zeta}{2 \sqrt{\mathrm{i} \beta \bar{L}}}+\sigma \frac{\sqrt{\mathrm{i} \beta \bar{L}}}{v}}(1-\sigma) \mathrm{d} \sigma .
$$

The second derivative of erfc is

$$
\frac{\mathrm{d}^{2}}{\mathrm{~d} z^{2}} \operatorname{erfc}(z)=\frac{4}{\sqrt{\pi}} z \mathrm{e}^{-z^{2}}
$$

This allows us to estimate

$$
\begin{aligned}
\left|R_{1}\right| & \leq \frac{4 \beta \bar{L}}{\sqrt{\pi}} \int_{0}^{1}\left(\frac{|\zeta|}{2 \sqrt{\beta \bar{L}}}+\sigma \frac{|\sqrt{\beta \bar{L}}|}{v}\right) \mathrm{e}^{\mp \sigma \zeta / v}(1-\sigma) \mathrm{d} \sigma \\
& \leq \frac{4 \beta \bar{L}}{\sqrt{\pi}} \mathrm{e}^{|\zeta| / v}\left(\frac{|\zeta|}{2 \sqrt{\beta \bar{L}}}+\mathrm{O}\left(v^{-1}\right)\right) \\
& =\mathrm{O}(\sqrt{v}) \quad \text { as } v \rightarrow \infty,
\end{aligned}
$$

uniformly for $|\zeta|<\sqrt{v}$. Substituting (26) and (27) into (23) gives

$$
I_{2}(\beta, \zeta)=1-\frac{2 \sqrt{\mathrm{i} \beta \bar{L}}}{v \sqrt{\pi}} \exp \left(\frac{-\zeta^{2}}{4 \mathrm{i} \beta \bar{L}}\right)-\frac{\zeta}{v} \operatorname{erf}\left(\frac{\zeta}{2 \sqrt{\mathrm{i} \beta \bar{L}}}\right)+\frac{R_{2}(\zeta)}{v^{2}},
$$

where $R_{2}$ is a combination of the errors in (26) and (28). So

$$
\left|R_{2}(\zeta)\right|=\mathrm{O}\left(1+\zeta^{2}\right) \quad \text { as } v \rightarrow \infty
$$

We thus have that

$$
\begin{aligned}
\frac{1}{\sqrt{v}} \mid \frac{2 \sqrt{\mathrm{i} \beta \bar{L}}}{\sqrt{\pi}} & \exp \left(\frac{-\zeta^{2}}{4 \mathrm{i} \beta \bar{L}}\right)+\zeta \operatorname{erf}\left(\frac{\zeta}{2 \sqrt{\mathrm{i} \beta \bar{L}}}\right)-\frac{R_{2}(\zeta)}{v} \mid \\
& \leq \frac{2 \sqrt{\beta \bar{L}}}{\sqrt{\pi v}}+\frac{|\zeta|}{\sqrt{v}}+\frac{\left|R_{2}\right|}{v^{3 / 2}} \\
& <2 \quad \text { for all } \zeta \in[-\sqrt{v}, \sqrt{v}] \text { and } v \text { sufficiently large. }
\end{aligned}
$$


We are now in a position to understand the asymptotics of $\left(I_{2}(\beta, \zeta)\right)^{v-1}$. We first consider

$$
\ln \left(1-\frac{a}{v}\right)^{v-1}=(v-1)\left(-\frac{a}{v}+\frac{R_{3}(a)}{v^{2}}\right)
$$

where

$$
\left|R_{3}(a)\right| \leq \frac{|a|^{2}}{1-|a| / v}
$$

provided that $|a|<v$. We take

$$
a=\frac{2 \sqrt{\mathrm{i} \beta \bar{L}}}{\sqrt{\pi}} \exp \left(\frac{-\zeta^{2}}{4 \mathrm{i} \beta \bar{L}}\right)+\zeta \operatorname{erf}\left(\frac{\zeta}{2 \sqrt{\mathrm{i} \beta \bar{L}}}\right)+\frac{R_{2}(\zeta)}{v}
$$

which satisfies $|a| / \sqrt{v}<2$ for $v$ sufficiently large by (31) and $a=\mathrm{O}(|\zeta|)$ as $|\zeta| \rightarrow \infty$ for $|\zeta|<\sqrt{v}$ by (30). We see that

$$
\frac{\left|R_{3}(a)\right|}{v}=\mathrm{O}\left(|a|^{2} / v\right) \quad \text { as } v \rightarrow \infty
$$

so exponentiation of (32) gives

$$
\left(1-\frac{a}{v}\right)^{v-1}=\mathrm{e}^{-a}\left(1+\mathrm{O}\left(v^{-1 / 2}\right)\right)\left(1+\mathrm{O}\left(\left(1+\zeta^{2}\right) / v\right)\right)
$$

where the error estimates are uniform for $|\zeta|<\sqrt{v}$ as $v \rightarrow \infty$ and

$$
\mathrm{e}^{-a}=\exp \left(-\frac{2 \sqrt{\mathrm{i} \beta \bar{L}}}{\sqrt{\pi}} \exp \left(\frac{-\zeta^{2}}{4 \mathrm{i} \beta \bar{L}}\right)-\zeta \operatorname{erf}\left(\frac{\zeta}{2 \sqrt{\mathrm{i} \beta \bar{L}}}\right)\right)\left[1+\mathrm{O}\left(\left(1+\zeta^{2}\right) / v\right)\right] .
$$

We can also write

$$
I_{1}(\beta, \zeta)=\frac{v}{\sqrt{\pi}} \frac{1}{\sqrt{\mathrm{i} \beta \bar{L}}} \exp \left(\frac{-\zeta^{2}}{4 \mathrm{i} \beta \bar{L}}\right)\left(1+\mathrm{O}\left(v^{-2}\right)\right) .
$$

Thus, taking (34) together with (29) and (33) gives the required estimate.

Lemma 4. Let $|\zeta|>\sqrt{v}$, then

$$
\left|I_{1}(\beta, \zeta)\left(I_{2}(\beta, \zeta)\right)^{v-1}\right| \leq \frac{1}{\pi}\left(\frac{\beta \bar{L}}{v^{2}}+\frac{\zeta^{2}}{\beta \bar{L}}\right)^{-1}+\mathrm{O}\left(v \zeta^{-3}\right) \quad \text { as }|\zeta| \rightarrow \infty .
$$

Proof. We make use of the asymptotic expression

$$
\operatorname{erfc}(z)=\frac{1}{\sqrt{\pi}} \mathrm{e}^{-z^{2}}\left(\frac{1}{z}+\mathrm{O}\left(z^{-3}\right)\right)
$$

as $z \rightarrow \infty$, valid for $|\arg z|<3 \pi / 4$. We see that

$$
\begin{aligned}
\mathrm{e}^{\zeta / v} \operatorname{erfc}\left(\frac{\zeta}{2 \sqrt{\mathrm{i} \beta \bar{L}}}\right. & \left.+\frac{\sqrt{\mathrm{i} \beta \bar{L}}}{v}\right)=\frac{1}{\sqrt{\pi}} \exp \left(\frac{-\zeta^{2}}{4 \mathrm{i} \beta \bar{L}}+\frac{\mathrm{i} \beta \bar{L}}{v^{2}}\right) \\
\times & {\left[\left(\frac{\zeta}{2 \sqrt{\mathrm{i} \beta \bar{L}}}+\frac{\sqrt{\mathrm{i} \beta \bar{L}}}{v}\right)^{-1}+\mathrm{O}\left(\zeta^{-3}\right)\right] . }
\end{aligned}
$$


Similarly,

$$
\begin{aligned}
\mathrm{e}^{-\zeta / v} \operatorname{erfc}\left(\frac{-\zeta}{2 \sqrt{\mathrm{i} \beta \bar{L}}}\right. & \left.+\frac{\sqrt{\mathrm{i} \beta \bar{L}}}{v}\right)=\frac{1}{\sqrt{\pi}} \exp \left(\frac{-\zeta^{2}}{4 \mathrm{i} \beta \bar{L}}+\frac{\mathrm{i} \beta \bar{L}}{v^{2}}\right) \\
\times & {\left[\left(\frac{-\zeta}{2 \sqrt{\mathrm{i} \beta \bar{L}}}+\frac{\sqrt{\mathrm{i} \beta \bar{L}}}{v}\right)^{-1}+\mathrm{O}\left(\zeta^{-3}\right)\right] . }
\end{aligned}
$$

Adding these gives the estimate

$$
\left|I_{2}(\beta, \zeta)\right| \leq \frac{\sqrt{\beta \bar{L}}}{v \sqrt{\pi}}\left(\frac{\beta \bar{L}}{v^{2}}+\frac{\zeta^{2}}{\beta \bar{L}}\right)^{-1}+\mathrm{O}\left(\zeta^{-3}\right) \quad \text { as }|\zeta| \rightarrow \infty .
$$

Taking (35) together with the estimates $\left|I_{2}(\beta, \zeta)\right| \leq 1$ and

$$
\left|I_{1}(\beta, \zeta)\right| \leq \frac{v}{\sqrt{\pi \beta \bar{L}}}
$$

gives us the estimate we require.

Proposition 2. With the notation above,

$\lim _{v \rightarrow \infty} \frac{1}{2 v} \int_{-\infty}^{\infty} I_{1}(\beta, \zeta)\left(I_{2}(\beta, \zeta)\right)^{v-1} \mathrm{~d} \zeta=\frac{1}{2 \sqrt{\pi}} \int_{-\infty}^{\infty} \frac{1}{\sqrt{\mathrm{i} \beta \bar{L}}} \exp \left(\frac{-\zeta^{2}}{4 \mathrm{i} \beta \bar{L}}\right) M(\beta, \zeta) \mathrm{d} \zeta$.

Proof. We split the region of integration as follows

$$
\begin{aligned}
& \frac{1}{2 v} \int_{-\infty}^{\infty} I_{1}(\beta, \zeta)\left(I_{2}(\beta, \zeta)\right)^{v-1} \mathrm{~d} \zeta=\frac{1}{2 v} \int_{-\sqrt{v}}^{\sqrt{v}} I_{1}(\beta, \zeta)\left(I_{2}(\beta, \zeta)\right)^{v-1} \mathrm{~d} \zeta \\
&+\frac{1}{2 v} \int_{|\zeta|>\sqrt{v}} I_{1}(\beta, \zeta)\left(I_{2}(\beta, \zeta)\right)^{v-1} \mathrm{~d} \zeta
\end{aligned}
$$

The function $M$ is bounded and satisfies

$$
|M(\beta, \zeta)| \leq \exp \left(\frac{2 \sqrt{\beta \bar{L}}}{\sqrt{\pi}}-\frac{|\zeta|}{2}\right)
$$

for $\zeta$ sufficiently large. Integrating (24) gives

$$
\begin{aligned}
\frac{1}{2 v} \int_{-\sqrt{v}}^{\sqrt{v}} I_{1}(\beta, \zeta)\left(I_{2}(\beta, \zeta)\right)^{v-1} \mathrm{~d} \zeta & =\frac{1}{2 \sqrt{\pi}} \int_{-\sqrt{v}}^{\sqrt{v}} \frac{1}{\sqrt{\mathrm{i} \beta \bar{L}}} \exp \left(\frac{-\zeta^{2}}{4 \mathrm{i} \beta \bar{L}}\right) M(\beta, \zeta) \mathrm{d} \zeta \\
+\mathrm{O} & {\left[\int_{-\sqrt{v}}^{\sqrt{v}} \exp \left(-\frac{|\zeta|}{2}\right)\left(\frac{1}{\sqrt{v}}+\frac{\zeta^{2}}{v}\right) \mathrm{d} \zeta\right] }
\end{aligned}
$$

and the integral in the remainder term converges as we let $v \rightarrow \infty$.

To deal with the integral

$$
\frac{1}{v} \int_{|\zeta|>\sqrt{v}} I_{1}(\beta, \zeta)\left(I_{2}(\beta, \zeta)\right)^{v-1} \mathrm{~d} \zeta
$$


we make use of the result of lemma 4 giving

$$
\begin{aligned}
\left|\frac{1}{v} \int_{|\zeta|>\sqrt{v}} I_{1}(\beta, \zeta)\left(I_{2}(\beta, \zeta)\right)^{v-1} \mathrm{~d} \zeta\right| & \\
& \leq \frac{1}{\pi} \int_{|\zeta|>\sqrt{v}}\left(\frac{\beta \bar{L}}{v^{2}}+\frac{\zeta^{2}}{\beta \bar{L}}\right)^{-1} \mathrm{~d} \zeta+\mathrm{O}\left(v^{-1}\right) \\
& \rightarrow 0 \quad \text { as } v \rightarrow \infty .
\end{aligned}
$$

Hence substituting (37) and (38) into (36) and taking the limit $v \rightarrow \infty$ gives the required result.

4.3. Properties of $M(\beta, \zeta)$. We wish to rotate the variable $\zeta$. However, we need to check that the function $M$ does not blow up for large $|\zeta|$.

If $\zeta=R \mathrm{e}^{\mathrm{i} \theta}$, then

$$
\operatorname{erf}\left(\frac{\zeta}{2 \sqrt{\mathrm{i} \beta \bar{L}}}\right)=\operatorname{erf}\left(\frac{R}{2 \sqrt{\beta \bar{L}}} \mathrm{e}^{\mathrm{i}(\theta-\pi / 4)}\right)=1+\mathrm{O}\left(R^{-1}\right)
$$

as $R \rightarrow \infty$, provided that $0<\theta<\pi / 2$ [1]. So,

$$
\begin{aligned}
\left|\exp \left(-\zeta \operatorname{erf}\left(\frac{\zeta}{2 \sqrt{\mathrm{i} \beta \bar{L}}}\right)\right)\right| & =\exp \left(-R \mathfrak{R e}\left[\mathrm{e}^{\mathrm{i} \theta} \operatorname{erf}\left(\frac{\zeta}{2 \sqrt{\mathrm{i} \beta \bar{L}}}\right)\right]\right) \\
& =\mathrm{e}^{-R \cos \theta} \mathrm{O}(1) \\
& \rightarrow 0 \quad \text { as } R \rightarrow \infty \text { provided } 0<\theta<\pi / 2
\end{aligned}
$$

and convergence is exponentially fast. Similarly,

$$
\begin{aligned}
\left|\exp \left(-\frac{2 \sqrt{\mathrm{i} \beta \bar{L}}}{\sqrt{\pi}} \exp \left(-\frac{\zeta^{2}}{4 \mathrm{i} \beta \bar{L}}\right)\right)\right|= & \exp \left(\frac{-2 \sqrt{\beta \bar{L}}}{\sqrt{\pi}} \exp \left(-\frac{R^{2}}{4 \beta \bar{L}} \sin 2 \theta\right)\right. \\
& \left.\times \cos \left(\frac{R^{2}}{4 \beta \bar{L}} \cos 2 \theta+\frac{\pi}{4}\right)\right) \\
\rightarrow & 1 \quad \text { as } R \rightarrow \infty
\end{aligned}
$$

provided $0<\theta<\pi / 2$.

Hence, if $0<\theta<\pi / 4$ then

$$
\lim _{R \rightarrow \pm \infty}\left|R M\left(\beta, R \mathrm{e}^{\mathrm{i} \theta}\right)\right|=0
$$

and we can make the change of variables $\zeta=\xi \sqrt{i \beta \bar{L}}$ :

$$
\int_{-\infty}^{\infty} \frac{1}{\sqrt{\mathrm{i} \beta \bar{L}}} \exp \left(\frac{-\zeta^{2}}{4 \mathrm{i} \beta \bar{L}}\right) M(\beta, \zeta) \mathrm{d} \zeta=\int_{-\infty}^{\infty} \mathrm{e}^{-\xi^{2} / 4} M(\beta, \xi \sqrt{\mathrm{i} \beta \bar{L}}) \mathrm{d} \xi
$$


We observe that

$$
\begin{aligned}
M(\beta, \xi \sqrt{\mathrm{i} \beta \bar{L}}) & =\exp \left(-\frac{2}{\sqrt{\pi}} \sqrt{\mathrm{i} \beta \bar{L}} \mathrm{e}^{-\xi^{2} / 4}-\xi \sqrt{\mathrm{i} \beta \bar{L}} \operatorname{erf}\left(\frac{\xi}{2}\right)\right) \\
& =\exp (-\sqrt{\mathrm{i} \beta \bar{L}} m(\xi))
\end{aligned}
$$

where

$$
m(\xi):=\frac{2}{\sqrt{\pi}} \mathrm{e}^{-\xi^{2} / 4}+\xi \operatorname{erf}(\xi / 2) .
$$

4.4. Proof of theorem 4, $m$ satisfies the bound $m(\xi) \geq 2 / \sqrt{\pi}$, so $M(\beta, \xi \sqrt{\mathrm{i} \beta \bar{L}})$ is bounded for all $\beta$. By the Weierstrass $M$-test the integral in (40) is uniformly convergent and hence

$$
\lim _{v \rightarrow \infty} E_{v}(\beta)
$$

is a continuous function of $\beta$. We appeal, once again, to the continuity theorem for characteristic functions to deduce that the limiting density $P(y)$ exists and is given by

$$
\begin{aligned}
P(y) & =\frac{1}{4 \pi^{3 / 2}} \int_{-\infty}^{\infty} \int_{-\infty}^{\infty} \mathrm{e}^{-\xi^{2} / 4} M(\beta, \xi \sqrt{\mathrm{i} \beta \bar{L}}) \mathrm{e}^{\mathrm{i} \beta y} \mathrm{~d} \xi \mathrm{d} \beta \\
& =\frac{1}{2 \pi^{3 / 2}} \mathfrak{R e} \int_{0}^{\infty} \int_{-\infty}^{\infty} \mathrm{e}^{-\xi^{2} / 4} M(\beta, \xi \sqrt{\mathrm{i} \beta \bar{L}}) \mathrm{e}^{\mathrm{i} \beta y} \mathrm{~d} \xi \mathrm{d} \beta .
\end{aligned}
$$

The integrand is dominated by

$$
\exp \left(-\xi^{2} / 4-\sqrt{\pi \bar{L} \beta}\right)
$$

so Fubini's theorem allows us to switch the order of integration. We quote the standard integral

$$
\int_{0}^{\infty} \mathrm{e}^{a x+b \sqrt{x}} \mathrm{~d} x=-\frac{1}{a}-\frac{b}{2 a} \sqrt{\frac{\pi}{-a}} \exp \left(\frac{-b^{2}}{4 a}\right) \operatorname{erfc}\left(\frac{-b}{2 \sqrt{-a}}\right)
$$

valid for $\mathfrak{R e} a<0$ and use this to perform the $\beta$ integral in (41). This leads to the result

$$
P(y)=\frac{\sqrt{\bar{L}}}{4 \pi y^{3 / 2}} \mathfrak{R e} \int_{-\infty}^{\infty} \exp \left(-\frac{\xi^{2}}{4}-\frac{\bar{L} m(\xi)^{2}}{4 y}\right) m(\xi) \operatorname{erfc}\left(\frac{\sqrt{\bar{L}} m(\xi)}{2 \mathrm{i} y}\right) \mathrm{d} \xi,
$$

which reduces to the form given in the statement of the theorem upon noticing that $\mathfrak{R e}\{\operatorname{erfc}(\mathrm{i} \theta)\}=1$ for all $\theta \in \mathbb{R}$. 
5. Value distribution of the eigenfunctions in the limit $v \rightarrow \infty$

To prove theorem [ 6 we use a standard approximation argument. We introduce the smoothed $\delta$-function

$$
\delta_{\epsilon}(x):=\frac{1}{2 \pi} \int_{-\infty}^{\infty} \mathrm{e}^{-\epsilon|\beta|+\mathrm{i} \beta x} \mathrm{~d} \beta=\frac{\epsilon}{\pi\left(\epsilon^{2}+x^{2}\right)} .
$$

Proposition 3. Let $\tilde{Q}_{v}(\eta)$ be related to $Q_{v}(\eta)$ by

$$
\tilde{Q}_{v}(\eta):=\frac{1}{\eta^{2}} Q_{v}\left(\frac{1}{\eta}\right)
$$

For any fixed $\epsilon$,

$$
\lim _{v \rightarrow \infty} \int_{0}^{\infty} \delta_{\epsilon}\left(\eta-\eta^{\prime}\right) \tilde{Q}_{v}\left(\eta^{\prime}\right) \mathrm{d} \eta^{\prime}=\int_{0}^{\infty} \delta_{\epsilon}\left(\eta-\eta^{\prime}\right) \tilde{Q}\left(\eta^{\prime}\right) \mathrm{d} \eta^{\prime},
$$

uniformly for $\eta$ in compact intervals, where

$$
\tilde{Q}(\eta)=\frac{1}{2 \pi^{3 / 2} \eta} \mathfrak{I m} \int_{-\infty}^{\infty} \exp \left(-\frac{\xi^{2}}{4}-\frac{\bar{L} m(\xi)^{2}}{8 \eta}\right) \operatorname{erfc}\left(\frac{\sqrt{L} m(\xi)}{2 \sqrt{2 \eta} \mathrm{i}}\right) \mathrm{d} \xi .
$$

Proof. Let

$$
\tilde{Q}_{\epsilon, v}(\eta):=\int_{0}^{\infty} \delta_{\epsilon}\left(\eta-\eta^{\prime}\right) \tilde{Q}_{v}\left(\eta^{\prime}\right) \mathrm{d} \eta^{\prime}=\lim _{N \rightarrow \infty} \frac{1}{N} \sum_{n=1}^{N} \delta_{\epsilon}\left(\eta-\frac{1}{v^{2} A_{i}(n, \mathbf{L} ; v)}\right) .
$$

We use the identity

$$
\delta_{\epsilon}\left(\eta-\frac{A}{B}\right)=B \delta_{\epsilon|B|}(B \eta-A)
$$

with

$$
A=\frac{\bar{L}}{v^{2}} \sum_{j=1}^{v} \sec ^{2} k L_{j}
$$

and

$$
B=2 \sec ^{2} k L_{i} .
$$

Thus

$$
\begin{aligned}
\delta_{\epsilon}\left(\eta-\frac{\sum_{j} \bar{L} \sec ^{2} k L_{j}}{2 v^{2} \sec ^{2} k L_{i}}\right)= & \frac{2 \sec ^{2} k L_{i}}{\pi} \mathfrak{R e} \int_{0}^{\infty} \exp \left(\mathrm{i} \beta\left(2 \eta-\frac{\bar{L}}{v^{2}}+2 \mathrm{i} \epsilon\right) \sec ^{2} k L_{i}\right) \\
& \times \prod_{j \neq i} \exp \left(-\frac{\mathrm{i} \beta \bar{L}}{v^{2}} \sec ^{2} k L_{j}\right) \mathrm{d} \beta .
\end{aligned}
$$

Applying identity (15) to this function and following the method in section 5 leads to

$$
\begin{aligned}
\frac{2 \bar{L}}{\pi^{2} \bar{d} v} \mathfrak{R e} \int_{0}^{\infty} \int_{-\infty}^{\infty} I_{4}(\beta, \zeta) & \left(\left(I_{2}(\beta, \zeta)\right)^{v-1}\right. \\
& +(v-1) I_{3}(\beta, \zeta) I_{1}(\beta, \zeta)\left(I_{2}(\beta, \zeta)\right)^{v-2} \mathrm{~d} \zeta \mathrm{d} \beta=: \tilde{P}_{\epsilon}(\eta)
\end{aligned}
$$


where the new integrals are

$$
I_{3}(\beta, \zeta):=\frac{1}{\pi} \int_{0}^{\pi} \sec ^{2} x \exp \left(\mathrm{i} \beta\left(2 \eta-\frac{\bar{L}}{v^{2}}+2 \mathrm{i} \epsilon\right) \sec ^{2} x+\frac{\mathrm{i} \zeta}{v} \tan x\right) \mathrm{d} x
$$

and

$$
I_{4}(\beta, \zeta):=\frac{1}{\pi} \int_{0}^{\pi} \sec ^{4} x \exp \left(\mathrm{i} \beta\left(2 \eta-\frac{\bar{L}}{v^{2}}+2 \mathrm{i} \epsilon\right) \sec ^{2} x+\frac{\mathrm{i} \zeta}{v} \tan x\right) \mathrm{d} x .
$$

$I_{3}$ and $I_{4}$ converge uniformly in $\zeta$ and $\eta$. Integral $I_{3}$ closely resembles integral $I_{1}$.

$$
I_{3}(\beta, \zeta)=\frac{\mathrm{e}^{2 \mathrm{i} \beta \eta-2 \epsilon \beta}}{\sqrt{\beta} \sqrt{2 \pi \epsilon-2 \pi \mathrm{i} \eta}}+\mathrm{O}_{\epsilon}\left(v^{-1}\right) \quad \text { as } v \rightarrow \infty .
$$

By making the substitution $z=\tan x, I_{4}$ reduces to

$$
\begin{aligned}
I_{4}(\beta, \zeta) & =\frac{1}{\pi} \int_{-\infty}^{\infty}\left(1+z^{2}\right) \exp \left(\mathrm{i} \beta\left(2 \eta-\frac{\bar{L}}{v^{2}}+2 \mathrm{i} \epsilon\right)\left(1+z^{2}\right)+\frac{\mathrm{i} \zeta z}{v}\right) \mathrm{d} z \\
& =\mathrm{O}_{\epsilon}(1) \quad \text { as } v \rightarrow \infty .
\end{aligned}
$$

This estimate ensures that $\tilde{Q}_{\epsilon, v}(\eta)$ is dominated by the second term. Since $I_{3}$ is also bounded as $v \rightarrow \infty$, the analysis of proposition 2 holds and

$$
\begin{aligned}
& \lim _{v \rightarrow \infty} \tilde{Q}_{\epsilon, v}(\eta) \\
& =\frac{2}{\pi^{2}} \mathfrak{R e} \frac{1}{\sqrt{2 \epsilon-2 \mathrm{i} \eta}} \int_{0}^{\infty} \int_{-\infty}^{\infty} \frac{1}{\beta \sqrt{\mathrm{i} \bar{L}}} \exp \left(2 \mathrm{i} \beta \eta-\frac{\zeta^{2}}{4 \mathrm{i} \beta \bar{L}}-2 \epsilon \beta\right) M(\beta, \zeta) \mathrm{d} \zeta \mathrm{d} \beta \\
& =\frac{2}{\pi^{2}} \mathfrak{R e}\left[\frac{1}{\sqrt{2 \epsilon-2 \mathrm{i} \eta}} \int_{-\infty}^{\infty} \int_{0}^{\infty} \exp \left(-\frac{\xi^{2}}{4}-\sqrt{\mathrm{i} \beta \bar{L}} m(\xi)+2 \mathrm{i} \beta \eta-2 \epsilon \beta\right) \frac{\mathrm{d} \beta}{\sqrt{\beta}} \mathrm{d} \xi\right],
\end{aligned}
$$

where we have made, once again, the substitution $\zeta=\xi \sqrt{\mathrm{i} \beta \bar{L}}$ in the final line. Putting $\theta^{2}=\beta$ reduces the $\beta$ integral to

$$
\begin{aligned}
& \int_{0}^{\infty} \mathrm{e}^{-\sqrt{\mathrm{i}} \bar{L} \theta m(\xi)-(2 \epsilon-2 \mathrm{i} \eta) \theta^{2}} \mathrm{~d} \theta \\
&=\frac{\sqrt{\pi}}{2 \sqrt{2 \epsilon-2 \mathrm{i} \eta}} \exp \left(\frac{-\bar{L} m(\xi)^{2}}{8(\eta+\mathrm{i} \epsilon)}\right) \operatorname{erfc}\left(\frac{\sqrt{\bar{L}} m(\xi)}{2 \mathrm{i} \sqrt{2 \eta+2 \mathrm{i} \epsilon}}\right),
\end{aligned}
$$

so

$$
\begin{aligned}
& \lim _{v \rightarrow \infty} \tilde{Q}_{\epsilon, v}(\eta) \\
& \quad=\frac{1}{2 \pi^{3 / 2}} \mathfrak{I m}\left[\frac{1}{\eta+\mathrm{i} \epsilon} \int_{-\infty}^{\infty} \exp \left(-\frac{\xi^{2}}{4}-\frac{\bar{L} m(\xi)^{2}}{8(\eta+\mathrm{i} \epsilon)}\right) \operatorname{erfc}\left(\frac{\sqrt{\bar{L}} m(\xi)}{2 \mathrm{i} \sqrt{2 \eta+2 \mathrm{i} \epsilon}}\right) \mathrm{d} \xi\right] \\
& \quad=\int_{0}^{\infty} \delta_{\epsilon}\left(\eta-\eta^{\prime}\right) \tilde{Q}\left(\eta^{\prime}\right) \mathrm{d} \eta^{\prime}
\end{aligned}
$$


We note that $\tilde{Q}(\eta)$ is a continuous probability density on $(0, \infty)$.

Proof of theorem [6] Let $0<a<b$ be fixed. Then let

$$
\mathbb{1}_{[a, b]}^{\epsilon}(\eta):=\int_{a}^{b} \delta_{\epsilon}(\eta-y) \mathrm{d} y
$$

$\mathbb{1}_{[a, b]}^{\epsilon}(\eta)$ converges pointwise as $\epsilon \rightarrow 0$ to the function $\mathbb{1}_{[a, b]}(\eta)$ everywhere apart from at the end-points $a$ and $b$. Given $\sigma>0$, consider the function

$$
\chi_{1}(\eta):=\mathbb{1}_{[a-\sigma, b+\sigma]}^{\epsilon}(\eta)+\sigma
$$

There exists $\epsilon>0$ such that:-

$-0 \leq \chi_{1}(\eta) \leq 2 \sigma$ for $\eta \leq a-2 \sigma$ and $\eta \geq b+2 \sigma$,

$-1 \leq \chi_{1}(\eta) \leq 1+\sigma$ for $a \leq \eta \leq b$,

$-0 \leq \chi_{1}(\eta) \leq 1+\sigma$ for $a-2 \sigma \leq \eta \leq a$ and $b \leq \eta \leq b+2 \sigma$.

This construction is illustrated in figure 4

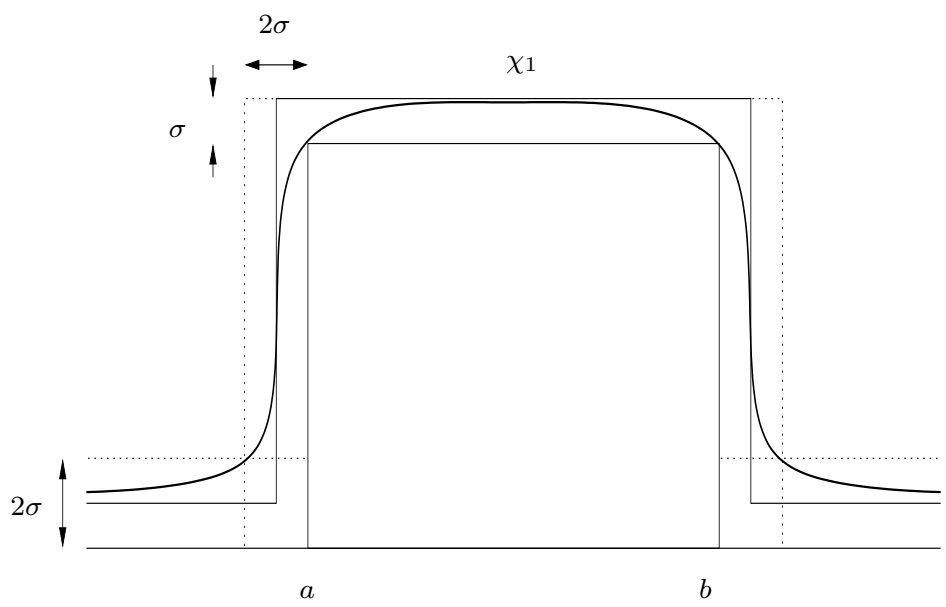

Fig. 4. Approximating $\mathbb{1}_{[a, b]}$ from above

Similarly, the function

$$
\chi_{2}(\eta)=\mathbb{1}_{[a+\sigma, b-\sigma]}^{\epsilon}(\eta)-\sigma
$$

satisfies for $\epsilon$ sufficiently small:-

- $-\sigma \leq \chi_{2}(\eta) \leq 0$ for $\eta \leq a$ and $\eta \geq b$,

$-1-2 \sigma \leq \chi_{2}(\eta) \leq 1$ for $a+2 \sigma \leq \eta \leq b-2 \sigma$,

- $-\sigma \leq \chi_{2}(\eta) \leq 1$ for all $a \leq \eta \leq a+2 \sigma$ and $b-2 \sigma \leq \eta \leq b$. 
So that for all $\eta \in[0, \infty)$

$$
\chi_{2}(\eta)<\mathbb{1}_{[a, b]}(\eta)<\chi_{1}(\eta) .
$$

Also,

$$
\begin{aligned}
\int_{0}^{\infty}\left[\chi_{1}(\eta)-\chi_{2}(\eta)\right] \tilde{Q}(\eta) \mathrm{d} \eta \leq 3 \sigma \int_{0}^{\infty} \tilde{Q}(\eta) \mathrm{d} \eta & +(1+2 \sigma) \int_{a-2 \sigma}^{a+2 \sigma} \tilde{Q}(\eta) \mathrm{d} \eta \\
& +(1+2 \sigma) \int_{b-2 \sigma}^{b+2 \sigma} \tilde{Q}(\eta) \mathrm{d} \eta
\end{aligned}
$$

which can be made arbitrarily small because $\tilde{Q}$ is a continuous probability density. It follows from proposition 3 that

$$
\lim _{v \rightarrow \infty} \int_{0}^{\infty} \chi_{1}(\eta) \tilde{Q}_{v}(\eta) \mathrm{d} \eta=\int_{0}^{\infty} \chi_{1}(\eta) \tilde{Q}(\eta) \mathrm{d} \eta
$$

and similarly for $\chi_{2}$. Hence, we can use the argument of lemma 2 mutatis mutandis, to deduce that

$$
\lim _{v \rightarrow \infty} \int_{a}^{b} \tilde{Q}_{v}(\eta) \mathrm{d} \eta=\int_{a}^{b} \tilde{Q}(\eta) \mathrm{d} \eta
$$

Making the substitution $\eta \mapsto 1 / \eta$ then completes the proof of convergence.

Expanding the error function in (3) as

$$
\operatorname{erfc}\left(\frac{\sqrt{\bar{L} \eta} m(\xi)}{2 \mathrm{i} \sqrt{2}}\right)=\frac{1}{\sqrt{\pi}} \exp \left(\frac{\bar{L} \eta m(\xi)^{2}}{8}\right)\left(\frac{2 \mathrm{i} \sqrt{2}}{\sqrt{\bar{L} \eta} m(\xi)}+\mathrm{O}\left(\eta^{-3 / 2}\right)\right)
$$

where the implied constant does not depend on $\xi$, yields

$$
Q(\eta)=\frac{b}{\eta^{3 / 2}}+\mathrm{O}\left(\eta^{-5 / 2}\right) \quad \text { as } \eta \rightarrow \infty
$$

where the constant $b$ is

$$
b=\frac{\sqrt{2}}{\sqrt{L} \pi^{2}} \int_{-\infty}^{\infty} \frac{\mathrm{e}^{-\xi^{2} / 4}}{m(\xi)} \mathrm{d} \xi \approx \frac{0.348}{\sqrt{\bar{L}}} .
$$

The algebraic decay of $Q(\eta)$ is in contrast to the exponential decay of the $\chi_{1}^{2}$ density. 


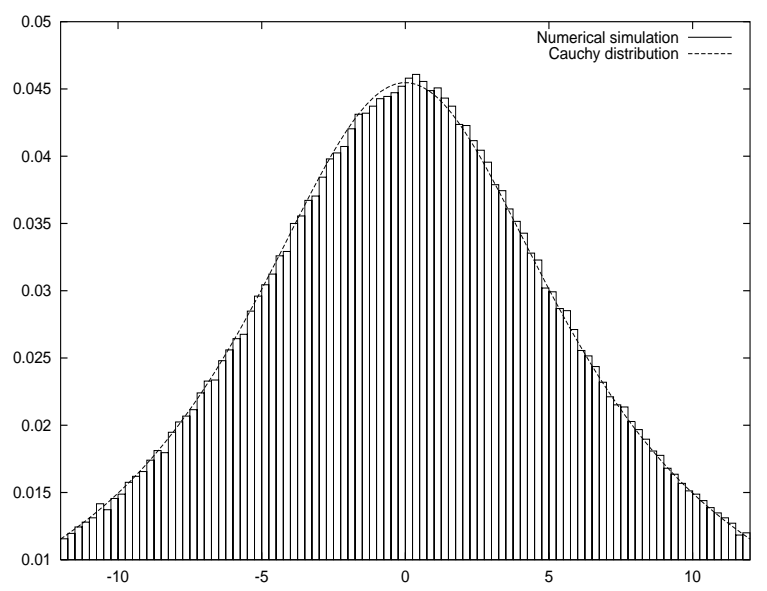

Fig. 5. The value distribution for the spectral determinant

\section{Numerical Results}

The results presented above show close agreement with numerical computations. We present these computations now by way of illustration.

In all the figures in this section, the choice of $\bar{L}=2$ has been made.

Figure 5 shows a comparison between a numerical evaluation of values taken by the spectral determinant and the Cauchy distribution. The numerical evaluation was based on a star graph with 7 randomly chosen bond lengths, and 100,000 samples of $k$.

Figure 6 shows a comparison between the distribution of values taken by the derivative of the spectral determinant at its zeros, and the corresponding numerical evaluation. Plotted is numerical data for a 70-bond star graph, together with the $v \rightarrow \infty$ limiting density given in theorem 4 Once again we see good agreement.

In figure 7 we compare a numerical evaluation of the density of values taken by the maximum norm of eigenvectors of a 50-bond graph to the $v \rightarrow \infty$ limiting density given in theorem [6] Also plotted for comparison is the density of the $\chi_{1}^{2}$ distribution associated with the $\mathrm{COE}$ of random matrices.

\section{Connections with the Šeba Billiard}

The correspondence between the spectral statistics of quantum star graphs and those of Šeba billiards with periodic boundary conditions has already been noted [3]. This is due to the fact that the spectral determinant for the star graphs (11) may be re-written in a form similar to the spectral determinant of a Šeba billiard:

$$
Z_{\text {Seba }}(E)=\sum_{k=1}^{\infty} \frac{1}{E_{k}^{(0)}-E}
$$

where the $E_{k}^{(0)}$ are the energy levels of the unperturbed system. Both spectral determinants have infinitely many poles of first order, which separate the energy 


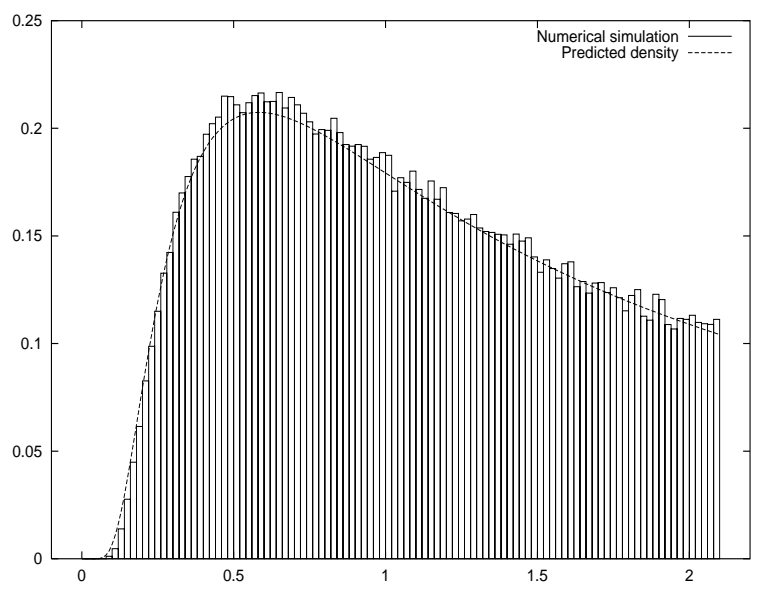

Fig. 6. The value distribution of $Z^{\prime}(k)$

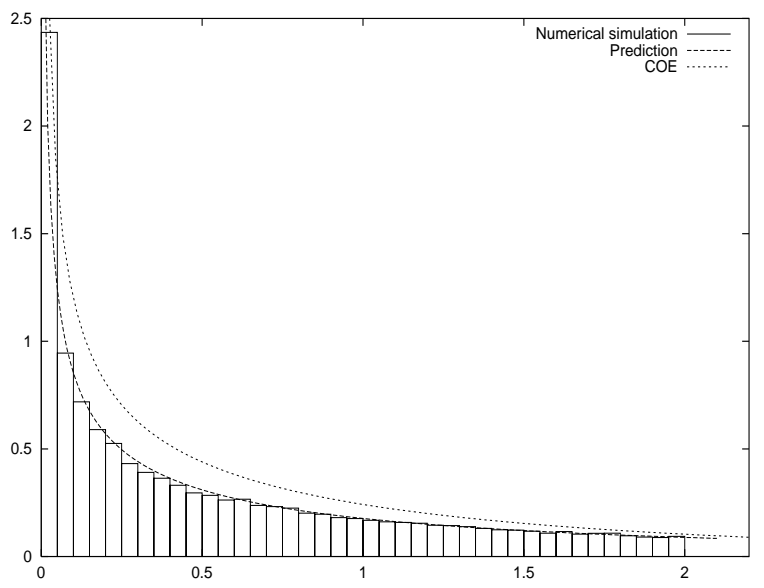

Fig. 7. The value distribution of $A_{i}(n, \mathbf{L} ; v)$

levels of the perturbed system. We therefore expect the value distribution of the spectral determinant of a Šeba billiard to be Cauchy to be consistent with theorems 1 and 2

This conjecture is supported by figure 8 which is a plot of the density of values given taken by the function

$$
\pi\langle d\rangle \sum_{k=1}^{K} \frac{1}{E_{k}^{(0)}-E}
$$

for $K=3000$ unperturbed levels of a rectangular quantum billiard with Neumann boundary conditions, with $E$ distributed uniformly between $E_{1000}^{(0)}$ and $E_{2000}^{(0)}$. The constant $\langle d\rangle$ is the mean density of levels of the system and it takes 
the place of the constant $v^{-1}$ in theorems 1 and 2 The fit to a Cauchy density is convincing.

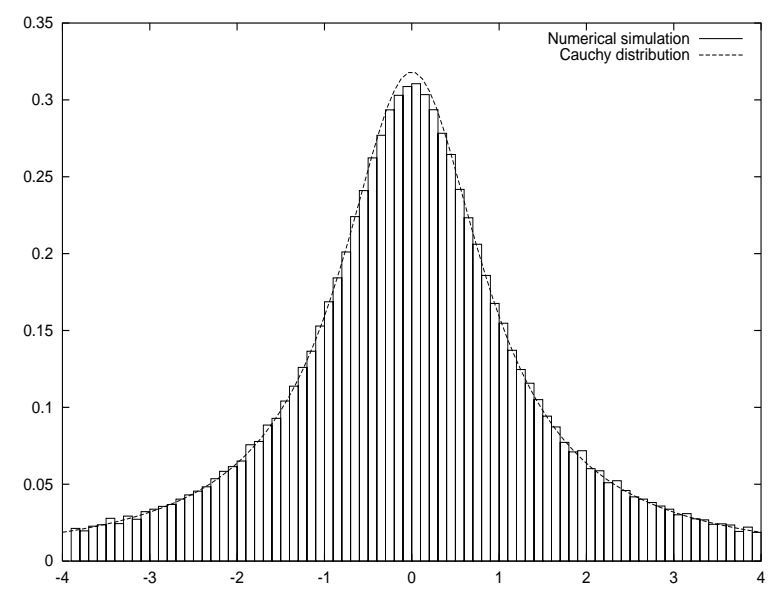

Fig. 8. The value distribution for the spectral determinant of a Šeba billiard

If we treat the unperturbed levels in (47) as independent identically distributed random variables with a uniform density then the random variables

$$
\frac{1}{E_{k}^{(0)}-E}
$$

have a distribution that falls into the domain of attraction of the stable Cauchy density. That the limiting density is Cauchy is then a classical result of probability theory [14.

We now present an argument which suggests that the normalisation constant associated with the wave functions of the Seba billiard also shares significant features with the normalisation constant of the star graphs (2).

The wave functions of a general Šeba billiard [29] can be written in the form

$$
\psi_{n}(\mathbf{x})=A_{n} \sum_{k=1}^{\infty} \frac{\psi_{k}^{(0)}\left(\mathbf{x}_{0}\right) \psi_{k}^{(0)}(\mathbf{x})}{E_{k}^{(0)}-E_{n}}
$$

where $E_{n}$ is the $n^{\text {th }}$ energy level and $E_{k}^{(0)}$ and $\psi_{k}^{(0)}$ are, respectively, the energy levels and wave functions of the original integrable system of which the Šeba problem is a perturbation. The Berry-Tabor Conjecture [7] asserts that the unperturbed levels $E_{k}^{(0)}$ are distributed like Poisson variables; that is independent and random. We fix the usual normalisation

$$
\int\left|\psi_{n}(\mathbf{x})\right|^{2} \mathrm{~d} \mathbf{x}=1
$$


which leads to a value for the constant $A_{n}$

$$
A_{n}^{2}=\left(\sum_{k=1}^{\infty} \frac{\left|\psi_{k}^{(0)}\left(\mathbf{x}_{0}\right)\right|^{2}}{\left(E_{k}^{(0)}-E_{n}\right)^{2}}\right)^{-1} .
$$

In the case that the unperturbed system is a rectangular quantum billiard with Neumann boundary conditions and sides of length $\alpha^{1 / 4}$ and $\alpha^{-1 / 4}$ the wavefunctions are

$$
\psi_{n, m}^{(0)}(x, y)=2 \cos \left(\frac{n \pi x}{\alpha^{1 / 4}}\right) \cos \left(m \pi y \alpha^{1 / 4}\right), \quad n, m=0,1,2, \ldots
$$

If we position the scatterer at the origin, then $\left|\psi_{n, m}^{(0)}\left(\mathbf{x}_{0}\right)\right|=2$. This billiard problem is equivalent to the billiard with periodic boundary conditions desymmetrised to remove degeneracies in the spectrum. Provided that the constant $\alpha$ satisfies certain diophantine conditions (see 23 13] for details) then $A_{n}^{2}$ in (49) is the reciprocal of a sum of functions with poles of second order distributed independently. These poles play the rôle of the singularities of the functions sec $k L_{j}$ which appear in the normalisation of the quantum graphs. Such poles determine the rate of decay of the tails of the relevant probability distributions, and this implies that the analysis performed in the present work also holds for this billiard problem. In particular, we conjecture that the distribution of the square of the $i^{\text {th }}$ coefficient of the eigenfunctions in the basis $\left|\psi_{k}^{(0)}\right\rangle$ is the same as the limiting distribution of $A_{i}(n, \mathbf{L} ; v)$.

We present in figure 9 the distribution of values taken by

$$
c \frac{\left(E_{i}^{(0)}-E_{n}\right)^{-2}}{\sum_{k=1}^{K}\left(E_{k}^{(0)}-E_{n}\right)^{-2}}
$$

where $n$ is now a random variable uniformly distributed on $\{1000, \ldots, 2000\}$ and we take $K=3000, i=1500$ and $\alpha=(\sqrt{5}-1) / 2$. The constant $c$, which in general may be expected to depend on $K$ and the distribution of $n$, is required to ensure that the sum of terms in the denominator is normalised and to compensate for the fact that the functions are not periodic. In order to compare with the corresponding results for star graphs we require that the tail of the distribution of $c\left(E_{i}^{(0)}-E_{n}\right)^{-2}$ is asymptotic to the tail of the distribution of $(2 / \bar{L}) \sec ^{2} k_{n} L_{i}$. Assuming $n$ to be distributed between $n_{\max }$ and $n_{\min }$, a heuristic examination of these densities leads to the association

$$
c=\frac{2}{\bar{L}}\left(E_{\max }-E_{\min }\right)^{2}\langle d\rangle^{2}
$$

where $E_{\max }$ and $E_{\min }$ are respectively the energy levels corresponding to $n=$ $n_{\max }$ and $n=n_{\min }$. For the data in figure 9 we get $c \approx 9.75 \times 10^{5}$.

\section{Acknowledgements}

JM is supported by an EPSRC Advanced Research Fellowship, the Nuffield Foundation (Grant NAL/00351/G), and the Royal Society (Grant 22355). BW is supported by an EPSRC studentship (Award Number 0080052X). Additionally, we are grateful for the financial support of the European Commission under the Research Training Network (Mathematical Aspects of Quantum Chaos) HPRNCT-2000-00103 of the IHP Programme. 


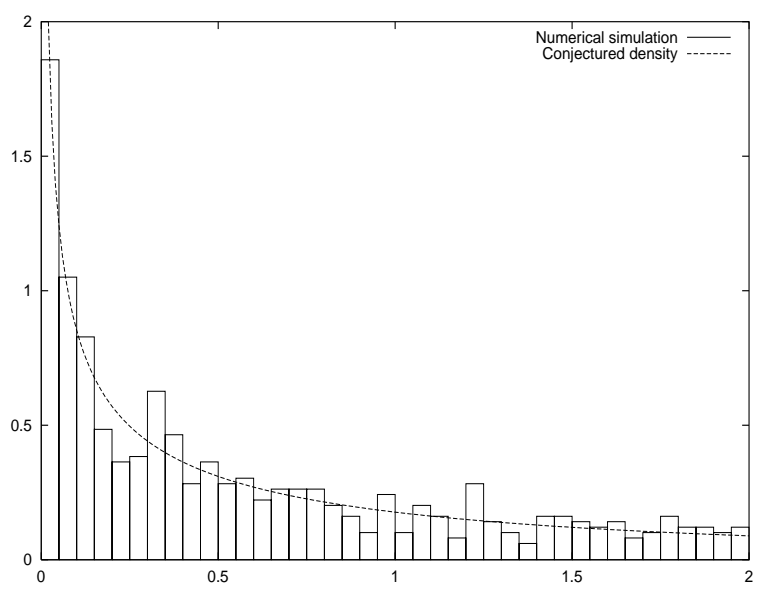

Fig. 9. The value distribution for the eigenfunctions of a Šeba billiard

\section{Appendix}

We here show that the distribution of the maximum amplitude $A_{i}(n, L ; v)$ completely determines the value distribution of the eigenfunctions on the $i^{\text {th }}$ bond, which is described by

$$
\frac{1}{N L_{i}} \sum_{n=1}^{N} \int_{0}^{L_{i}} f\left(\psi_{i}^{(n)}(x)\right) \mathrm{d} x
$$

where $f$ is an arbitrary bounded continuous function. Let us in fact consider the more general joint distribution,

$$
\frac{1}{N L_{i}} \sum_{n=1}^{N} \int_{0}^{L_{i}} F\left(\cos k_{n}\left(x-L_{i}\right), v^{2} A_{i}(n, L ; v)\right) \mathrm{d} x
$$

where $F$ is a bounded continuous function in two variables. We obtain the expression (51) for the choice $F(t, \eta)=f(t \sqrt{\eta})$ provided $f$ is even (which, as will become clear below, we may assume w.l.o.g.).

We begin with the special case when $F$ factorizes, i.e., $F(t, \eta)=f_{1}(t) f_{2}(\eta)$ where $f_{1}, f_{2}$ are arbitrary bounded continuous functions. Then

$$
\begin{aligned}
\frac{1}{L_{i}} \int_{0}^{L_{i}} f_{1}\left(\cos k_{n}\left(x-L_{i}\right)\right) \mathrm{d} x & =\int_{0}^{1} f_{1}(\cos (2 \pi x)) \mathrm{d} x+\mathrm{O}\left(k_{n}^{-1}\right) \\
& =\frac{1}{\pi} \int_{-1}^{1} f_{1}(t) \frac{\mathrm{d} t}{\sqrt{1-t^{2}}}+\mathrm{O}\left(k_{n}^{-1}\right),
\end{aligned}
$$

and, by theorem 5

$$
\frac{1}{N} \sum_{n=1}^{N} f_{2}\left(v^{2} A_{i}(n, L ; v)\right) \rightarrow \int_{0}^{\infty} f_{2}(\eta) Q_{v}(\eta) \mathrm{d} \eta
$$


as $N \rightarrow \infty$. Since the mean density of the eigenvalues $k_{n}$ is constant, we have $\sum_{n \leq N} \mathrm{O}\left(k_{n}^{-1}\right)=\mathrm{O}(\log N)$ and thus from (52) and (53)

$$
\begin{aligned}
\lim _{N \rightarrow \infty} \frac{1}{N L_{i}} \sum_{n=1}^{N} \int_{0}^{L_{i}} F\left(\cos k_{n}\left(x-L_{i}\right), v^{2} A_{i}(n, L ; v)\right) \mathrm{d} x \\
=\frac{1}{\pi} \int_{-1}^{1} \int_{0}^{\infty} F(t, \eta) Q_{v}(\eta) \frac{\mathrm{d} \eta \mathrm{d} t}{\sqrt{1-t^{2}}} .
\end{aligned}
$$

This holds for functions $F=f_{1} f_{2}$ and, by linearity, also for finite linear combinations of such functions. Given any $\epsilon>0$ we can approximate any bounded continuous $F$ from above and below by such finite linear combinations $F_{+}$and $F_{-}$, respectively, such that

$$
\frac{1}{\pi} \int_{-1}^{1} \int_{0}^{\infty}\left[F_{+}(t, \eta)-F_{-}(t, \eta)\right] Q_{v}(\eta) \frac{\mathrm{d} \eta \mathrm{d} t}{\sqrt{1-t^{2}}}<\epsilon .
$$

Since $\epsilon$ can be arbitrarily small, (54) holds in fact for any bounded continuous $F$.

We can therefore choose $F(t, \eta)=f(t \sqrt{\eta})$ as a test function, and we find

$$
\lim _{N \rightarrow \infty} \frac{1}{N L_{i}} \sum_{n=1}^{N} \int_{0}^{L_{i}} f\left(\psi_{i}^{(n)}(x)\right) \mathrm{d} x=\int_{-\infty}^{\infty} f(r) R_{v}(r) \mathrm{d} r
$$

with the limiting distribution

$$
R_{v}(r)=\frac{1}{\pi} \int_{r^{2}}^{\infty} Q_{v}(s) \frac{\mathrm{d} s}{\sqrt{s-r^{2}}}
$$

The limit $v \rightarrow \infty$ can be handled in an analogous way and leads to the same formulas for the limit $R(r)$ of $R_{v}(r)$ with $Q_{v}(s)$ replaced by $Q(s)$ in the above. It follows from the asymptotic expansion (44) that $R(r)$ also decays with an algebraic tail.

\section{References}

1. Abramowitz, M. and Stegun, I. A.:Handbook of mathematical functions. Dover Publishing, 1965

2. Barra, F. and Gaspard, P.: On the level spacing distribution in quantum graphs. J. Stat. Phys. 101, 283-319 (2000)

3. Berkolaiko, G., Bogomolny, E. B. and Keating, J. P.: Star graphs and Šeba billiards. J. Phys. A 34, 335-350 (2001)

4. Berkolaiko, G. and Keating, J. P.: Two-point spectral correlations for star graphs. J. Phys. A 32, 7827-7841 (1999)

5. Berkolaiko, G., Schanz, H. and Whitney, R. S.: Leading off-diagonal correction to the form factor of large graphs. Phys. Rev. Lett. 88, art. no. 104101 (2002)

6. Berry, M. V.: Regular and irregular semiclassical wavefunctions. J. Phys. A 10, 2083-2091 (1977)

7. Berry, M. V. and Tabor, M.: Level clustering in the regular spectrum. Proc. Roy. Soc. Lond. A 356, 375-394 (1977)

8. Bogomolny, E. B., Gerland, U. and Schmit, C.: Models of intermediate spectral statistics. Phys. Rev. E 59, R1315-R1318 (1999) 
9. Bogomolny, E. B., Gerland, U. and Schmit, C.: Singular statistics. Phys. Rev. E 63, art. no. 036206 (2001)

10. Bogomolny, E. B., Leboeuf, P. and Schmit, C.: Spectral statistics of chaotic systems with a pointlike scatterer. Phys. Rev. Lett. 85, 2486-2489 (2000)

11. Bolte, J. and Harrison, J.: Spectral statistics for the Dirac operator on graphs. J. Phys. A 36, 2747-2769 (2003)

12. Desbois, J.: Spectral determinant of Schrödinger operator on graphs. J. Phys. A 33, 63-67 (2000)

13. Eskin, A., Margulis, G. and Mozes, S.: Quadratic forms of signature $(2,2)$ and eigenvalue spacings on rectangular 2-tori. Preprint available at www.math.uchicago.edu/ ${ }^{\text {eskin/, }}$ 2001

14. Feller, W.: An introduction to probability theory and its applications. Wiley, 1971

15. Haake, F. and Życzkowski, K.: Random-matrix theory and eigenmodes of dynamical systems. Phys. Rev. A 42, 1013-1016 (1990)

16. Hughes, C. P., Keating, J. P. and O'Connell, N.: 2000 Random matrix theory and the derivative of the Riemann zeta function. Proc. Roy. Soc: A 456, 2611-2627 (2000)

17. Keating, J. P. and Snaith, N. C.: Random matrix theory and $\zeta(1 / 2+\mathrm{i} t)$. Commun. Math. Phys. 214 57-89 (2000)

18. Kottos, T. and Schanz, H.: Quantum graphs: a model for quantum chaos. Physica E 9, $523-530(2001)$

19. Kottos, T. and Smilansky, U.: Quantum Chaos on graphs Phys. Rev. Lett. 79, 4794-4797 (1997)

20. Kottos, T. and Smilansky, U.: Periodic orbit theory and spectral statistics for quantum graphs. Ann. Phys. 274, 76-124 (1999)

21. Kurasov, P. and Stenberg, F.: On the inverse scattering problem on branching graphs. J. Phys. A 35, 101-121 (2002)

22. Kurlberg, P. and Rudnick, Z.: Value distribution for eigenfunctions of desymmetrized quantum maps. Int. Math. Res. Notices 18, 985-1002 (2001)

23. Marklof, J.: Spectral form factors of rectangle billiards. Commun. Math. Phys. 199, 169$202(1998)$

24. Pakoński, P., Życzkowski, K. and Kuś, M.: Classical 1D maps, quantum graphs and ensembles of unitary matrices. J. Phys. A 34, 9303-9319 (2001)

25. Pascaud, M. and Montambaux, G.: Persistent currents on networks. Phys. Rev. Lett. 82, 4512-4515 (1999)

26. Schanz, H. and Smilansky, U.: Spectral statistics for quantum graphs: periodic orbits and combinatorics in Proceedings of the Australian summer school on quantum chaos and mesoscopics, Canberra 1999

27. Schanz, H. and Smilansky, U.: Periodic-orbit theory of Anderson localisation on graphs. Phys. Rev. Lett. 84, 1472-1430 (2000)

28. Šeba, P.: Wave chaos in singular quantum billiard. Phys. Rev. Lett. 64, 1855-1858 (1990)

29. Šeba, P. and Albeverio, S.: Wave chaos in quantum systems with point interaction. J. Stat. Phys 64, 369-383 (1991)

30. Tanner, G.: Unitary stochastic matrix ensembles and spectral statistics. J. Phys. A 34, 8485-8500 (2001)

31. Weyl, H.: Über die Gleichverteilung von Zahlen mod. Eins. Math. Ann. 77, 313-352 (1916) 